\title{
Strategic Design and Synthesis of Osmium(II) Complexes Bearing a Single Pyridyl Azolate $\pi$-Chromophore: Achieving High-Efficiency Blue Phosphorescence by Localized Excitation
}

\author{
Yi-Ming Cheng, Elise Y. Li, Gene-Hsiang Lee, and Pi-Tai Chou* \\ Department of Chemistry and Instrumentation Center, National Taiwan University, \\ Taipei 106, Taiwan
}

\section{Sue-Yi Lin and Ching-Fong Shu}

Department of Applied Chemistry, National Chiao Tung University, Hsinchu 300, Taiwan

Kwun-Chi Hwang, Yao-Lun Chen, Yi-Hwa Song, and Yun Chi

Department of Chemistry, National Tsing Hua University, Hsinchu 300, Taiwan

Received August 2, 2007

\begin{abstract}
We present the strategic design and synthesis of Os(II) complexes bearing a single pyridyl azolate $\pi$-chromophore with an aim to attain high efficiency blue phosphorescence by way of localized transition. It turns out that our proposal of localized excitation seems to work well upon anchoring a single $\pi$-chromophore on the Os(II) complexes such that the control of MLCT versus $\pi \pi^{*}$ (or even LLCT) transitions is more straightforward. Among the titled complexes, [Os $\left.(\mathrm{CO})_{3}(\mathrm{tfa})(\mathrm{fppz})\right](\mathbf{1})$ and $\left[\mathrm{Os}(\mathrm{CO})_{3}(\mathrm{tfa})(\mathrm{fbtz})\right](\mathbf{5})(\mathrm{tfa}=$ trifluoroacetate, $(\mathrm{fppz}) \mathrm{H}=3$-(trifluoromethyl)5-(2-pyridyl)pyrazole, and (fbtz)H = 3-(trifluoromethyl)-5-(4-tert-butyl-2-pyridyl)-1,2,4-triazole) give the anticipated blue phosphorescence with efficiencies of $0.26\left(\lambda_{\max }=460 \mathrm{~nm}\right)$ and $0.27\left(\lambda_{\max }=450 \mathrm{~nm}\right)$, respectively. For their halide analogues $\left[\mathrm{Os}(\mathrm{CO})_{3}(\mathrm{X})(\mathrm{fppz})\right](2, \mathrm{X}=\mathrm{Cl} ; \mathbf{3}, \mathrm{X}=\mathrm{Br} ; \mathbf{4}, \mathrm{X}=\mathrm{I})$ and phosphine-substituted isomeric derivatives $\left[\mathrm{Os}(\mathrm{tfa})(\mathrm{fppz})\left(\mathrm{PPh}_{2} \mathrm{Me}\right)_{2}(\mathrm{CO})\right](6-8)$, the localization of the excitation energy seems to populate at certain vibrational modes with weak bonding strength and hence an associated shallow potential energy surface to induce a facile radiationless transition. Furthermore, their ancillary ligands play an important role in fine-tuning not only the energy gap but also the emission intensity, i.e., in manifesting the radiationless transition pathways. Our results clearly show that there is always a tradeoff upon varying the parameters in an aim to optimize the hue and efficiency of phosphorescence toward blue.
\end{abstract}

\section{Introduction}

Luminescent complexes possessing third-row transition metal elements have received considerable research attention. ${ }^{1}$ In addition to their good thermal stability, these metal

* To whom correspondence should be addressed. E-mail: ychi@ mx.nthu.edu.tw (P.-T.C.), chop@ntu.edu.tw (Y.C.). Fax: +886 3-572 0864 (P.-T.C.), +886 2-2369 5208 (Y.C.).

(1) (a) Evans, R. C.; Douglas, P.; Winscom, C. J. Coord. Chem. Rev. 2006, 250, 2093. (b) Yersin, H. Top. Curr. Chem. 2004, 241, 1. (c) Lai, S.-W.; Che, C.-M. Top. Curr. Chem. 2004, 241, 27. (d) Holder, E.; Langeveld, B. M. W.; Schubert, U. S. Adv. Mater. 2005, 17, 1109. (e) Chou, P.-T.; Chi, Y. Chem.-Eur. J. 2007, 13, 380. (f) Browne, W. R.; O’Boyle, N. M.; McGarvey, J. J.; Vos, J. G. Chem. Soc. Rev. 2005, 34, 641 .

10276 Inorganic Chemistry, Vol. 46, No. 24, 2007 complexes often undergo unitary $\mathrm{S}_{1}-\mathrm{T}_{1}$ intersystem crossing and exhibit bright luminescence from the triplet manifold due to the enhanced spin-orbit coupling. Tuning of emission over the entire near-infrared (NIR) to visible spectra could be achieved by judicious modification of both a ligated chromophore and the nature of the central metal atom. ${ }^{2}$

(2) (a) Tung, Y.-L.; Lee, S.-W.; Chi, Y.; Chen, L.-S.; Shu, C.-F.; Wu, F.-I.; Carty, A. J.; Chou, P.-T.; Peng, S.-M.; Lee, G.-H. Adv. Mater. 2005, 17, 1059. (b) Tamayo, A. B.; Garon, S.; Sajoto, T.; Djurovich, P. I.; Tsyba, I. M.; Bau, R.; Thompson, M. E. Inorg. Chem. 2005, 44, 8723. (c) Hwang, F.-M.; Chen, H.-Y.; Chen, P.-S.; Liu, C.-S.; Chi, Y.; Shu, C.-F.; Wu, F.-I.; Chou, P.-T.; Peng, S.-M.; Lee, G.-H. Inorg. Chem. 2005, 44, 1344. (d) Matsushita, T.; Asada, T.; Koseki, S. J. Phys. Chem. C 2007, 111, 6897.

10.1021/ic7015269 CCC: $\$ 37.00 \quad$ C 2007 American Chemical Society Published on Web 10/20/2007 
However, there are only a few reports on the production of much higher energy, saturated blue or even near-UV phosphorescent materials. ${ }^{3}$ This is partly attributed to the fact that upon increasing the emission energy, there is a gradual decrease in admixture of the metal-to-ligand charge transfer (MLCT) transition, ${ }^{4}$ and the reduction of metal participation leads to the adverseness on spin-orbit coupling, such that the state mixing (first-order approximation) between singlet and triplet manifolds is less efficient. The net result reduces the $\mathrm{T}_{1}-\mathrm{S}_{0}$ transition moment, and the radiative decay rate constant $k_{\mathrm{r}}$ is decreased accordingly. Note the emission quantum efficiency, QY, is expressed as QY $=k_{\mathrm{r}} /\left(k_{\mathrm{r}}+k_{\mathrm{nr}}\right)$. Under identical nonradiative deactivation rate constant $k_{\mathrm{nr}}$, lowering the $k_{\mathrm{r}}$ value thus causes a decrease of the corresponding QY. Moreover, the increase of radiative lifetimes is undesirable for phosphorescent OLEDs, as it would cause saturation and triplet-triplet annihilation at higher current and driving voltage. ${ }^{5}$

With an aim to attain the saturated blue phosphors, we recently launched a venture project on preparation of a series of Os(II) carbonyl complexes with two identical pyridyl azolate chromophores. It was found that the geometrical isomers with cis-oriented azolate fragments exhibited much stronger room-temperature phosphorescence compared with the corresponding trans-counterparts. The results have been tentatively rationalized by the increased electrostatic repulsion in the trans-azolate arrangement, which caused shallow potential energy surface and hence increased radiationless deactivation rate constant. ${ }^{6,7}$ As for another observation, upon increase of the interligand $\pi \pi^{*}$ energy gap of chelating ligands in the trisubstituted pyridyl azolate- $\operatorname{Ir}(\mathrm{III})$ complexes, the lowest energy $\pi \pi^{*}$ state showed significant

(3) (a) Nazeeruddin, M. K.; Humphry-Baker, R.; Berner, D.; Rivier, S.; Zuppiroli, L.; Graetzel, M. J. Am. Chem. Soc. 2003, 125, 8790. (b) Coppo, P.; Plummer, E. A.; De Cola, L. Chem. Commun. 2004, 1774. (c) Yeh, S.-J.; Wu, W.-C.; Chen, C.-T.; Song, Y.-H.; Chi, Y.; Ho, M.-H.; Hsu, S.-F.; Chen, C.-H. Adv. Mater. 2005, 17, 285. (d) Li, J.; Djurovich, P. I.; Alleyne, B. D.; Yousufuddin, M.; Ho, N. N.; Thomas, J. C.; Peters, J. C.; Bau, R.; Thompson, M. E. Inorg. Chem. 2005, 44, 1713. (e) Lo, S.-C.; Shipley, C. P.; Bera, R. N.; Harding, R. E.; Cowley, A. R.; Burn, P. L.; Samuel, I. D. W. Chem. Mater. 2006, 18, 5119. (f) Chew, S.; Lee, C. S.; Lee, S.-T.; Wang, P.; He, J.; Li, W.; Pan, J.; Zhang, X.; Kwong, H. Appl. Phys. Lett. 2006, 88, 093510. (g) You, Y.; Kim, S. H.; Jung, H. K.; Park, S. Y. Macromolecules 2006, 39, 349. (h) Shih, P.-I.; Chien, C.-H.; Chuang, C.-Y.; Shu, C.F.; Yang, C.-H.; Chen, J.-H.; Chi, Y. J. Mater. Chem. 2007, 17, 1692. (i) Takizawa, S.; Nishida, J.; Tsuzuki, T.; Tokito, S.; Yamashita, Y. Inorg. Chem. 2007, 46, 4308. (j) Chen, L.; You, H.; Yang, C.; Ma, D.; Qin, J. Chem. Commun. 2007, 1352.

(4) (a) Sajoto, T.; Djurovich, P. I.; Tamayo, A.; Yousufuddin, M.; Bau, R.; Thompson, M. E.; Holmes, R. J.; Forrest, S. R. Inorg. Chem. 2005, 44, 7992. (b) Yang, C.-H.; Cheng, Y.-M.; Chi, Y.; Hsu, C.-J.; Fang, F.-C.; Wong, K.-T.; Chou, P.-T.; Chang, C.-H.; Tsai, M.-H.; Wu, C.C. Angew. Chem., Int. Ed. 2007, 46, 2418.

(5) (a) Yersin, H.; Donges, D. Top. Curr. Chem. 2001, 214, 81. (b) Connick, W. B.; Miskowski, V. M.; Houlding, V. H.; Gray, H. B. Inorg. Chem. 2000, 39, 2585. (c) Kavitha, J.; Chang, S.-Y.; Chi, Y.; Yu, J.-K.; Hu, Y.-H.; Chou, P.-T.; Peng, S.-M.; Lee, G.-H.; Tao, Y.T.; Chien, C.-H.; Carty, A. J. Adv. Funct. Mater. 2005, 15, 223. (d) Reineke, S.; Walzer, K.; Leo, K. Phys. Rev. B 2007, 75, 125328.

(6) (a) Wu, P.-C.; Yu, J.-K.; Song, Y.-H.; Chi, Y.; Chou, P.-T.; Peng, S.-M.; Lee, G.-H. Organometallics 2003, 22, 4938. (b) Yu, J.-K.; Hu, Y.-H.; Cheng, Y.-M.; Chou, P.-T.; Peng, S.-M.; Lee, G.-H.; Carty, A. J.; Tung, Y.-L.; Lee, S.-W.; Chi, Y.; Liu, C.-S. Chem.-Eur. J. 2004, 10, 6255.

(7) (a) Chou, P.-T.; Chi, Y. Eur. J. Inorg. Chem. 2006, 3319. (b) Chi, Y.; Chou, P.-T. Chem. Soc. Rev. 2007, 36, 1421. mixing with a thermally accessible ligand-to-ligand charge transfer (LLCT) state. ${ }^{8}$ This unusual state mixing then increases the radiative lifetime owing to its largely chargeseparated character and partially forbidden transition probability versus the ground state.

On the basis of the above progress, it seems that confinement of the electronic excitation to a single ligated chromophore may result in a possible increase of the emission efficiency. ${ }^{9}$ However, it may conversely cause an adverse effect in that the associated metal-ligand bond strength of the locally excited chromophore is weaker than those metal complexes possessing multiple isoenergetic chromophores such that the excitation energy can be randomly dissipated. Consequently, the presence of weak bonds may induce rapid radiationless deactivation and then offset the presuming gain of emission efficiency upon the energy localization (vide supra).

Unfortunately, at the current stage, the theoretical assessment of radiationless deactivation for the sophisticated molecules like transition metal complexes is still formidable. It is thus desirable to construct a series of prototypical systems, for which the direct photoexcitation is preliminarily associated with a single $\pi$-chromophore, in an aim to examine the fundamental properties elaborated above. For a comparison, the ligand-dependent excited-state behaviors of the related $\operatorname{Re}(\mathrm{I})$ and $\mathrm{Ru}(\mathrm{II})$ systems were documented, ${ }^{10}$ offering certain prospective molecular designs for various functional photonic materials, such as sensitizers, luminophores, and even luminescent sensors.

\section{Experimental Section}

General Information and Materials. All reactions were performed under a nitrogen atmosphere using anhydrous solvents or solvents treated with an appropriate drying reagent. Mass spectra were obtained on a JEOL SX-102A instrument operating in electron impact (EI) mode or fast atom bombardment (FAB) mode. ${ }^{1} \mathrm{H}$ and ${ }^{19} \mathrm{~F}$ NMR spectra were recorded on Varian Mercury-400 or INOVA500 instruments. Elemental analyses were conducted at the NSC Regional Instrumentation Center at National Chiao Tung University. Osmium reagent $\left[\mathrm{Os}(\mathrm{CO})_{3}(\mathrm{tfa})_{2}\right]$ was prepared from the direct treatment of $\mathrm{Os}_{3}(\mathrm{CO})_{12}$ with trifluoroacetic acid. ${ }^{18}$ The chelating ligands 3-(trifluoromethyl)-5-(2-pyridyl)pyrazole (fppz)H and 3-(trifluoromethyl)-5-(4-tert-butyl-2-pyridyl)-1,2,4-triazole (fbtz)H were prepared according to the methods reported in the literature. ${ }^{11}$

Steady-state absorption and emission spectra were recorded with a Hitachi (U-3310) spectrophotometer and an Edinburgh (FS920) fluorometer, respectively. Quinine sulfate with an emission yield of $\Phi \sim 0.57$ in $1.0 \mathrm{~N} \mathrm{H}_{2} \mathrm{SO}_{4}$ served as the standard to calculate the emission quantum yield. The emission decays of $<10 \mu$ s were measured by an Edinburgh (FS920) fluorometer using a time-

(8) (a) Yang, C.-H.; Li, S.-W.; Chi, Y.; Cheng, Y.-M.; Yeh, Y.-S.; Chou, P.-T.; Lee, G.-H.; Wang, C.-H.; Shu, C.-F. Inorg. Chem. 2005, 44, 7770. (b) Yeh, Y.-S.; Cheng, Y.-M.; Chou, P.-T.; Lee, G.-H.; Yang, C.-H.; Chi, Y.; Shu, C.-F.; Wang, C.-H. Chem. Phys. Chem. 2006, 7, 2294.

(9) Chang, C.-J.; Yang, C.-H.; Chen, K.; Chi, Y.; Shu, C.-F.; Ho, M.-L.; Yeh, Y.-S.; Chou, P. T. Dalton Trans. 2007, 1881

(10) (a) Stufkens, D. J.; Vlcek, A., Jr. Coord. Chem. Rev. 1998, 177, 127. (b) Vlček, A., Jr.; Záliš, S. Coord. Chem. Rev. 2007, 251, 258.

(11) (a) Thiel, W. R.; Eppinger, J. Chem.-Eur. J. 1997, 3, 696. (b) Satake, A.; Nakata, T. J. Am. Chem. Soc. 1998, 120, 10391. 
Cheng et al.

correlated single photon counting technique. The data were analyzed by the sum of exponential functions, which allows partial removal of the instrument time broadening and consequently renders a temporal resolution of $\sim 200 \mathrm{ps}$. The long-lived ( $\geq 10 \mu \mathrm{s})$ phosphorescence spectra were measured by an ultrasensitive detection system coupled with a laser excitation source. Briefly, an Nd:YAG (355 nm, 8 ns, Continuum Surelite II) pumped optical parametric oscillator coupled with a second harmonic device served as a tunable excitation source. The resulting emission was detected with an intensified charge coupled detector (ICCD, Princeton Instruments, model $576 \mathrm{G} / 1$ ). Data were analyzed using the nonlinear leastsquares procedure in combination with an iterative convolution method.

Synthesis of $\left[\mathrm{Os}(\mathbf{C O})_{3}(\mathbf{t f a})(\mathbf{f p p z})\right](\mathbf{1}) .\left[\mathrm{Os}(\mathrm{CO})_{3}(\mathrm{tfa})_{2}\right](70 \mathrm{mg}$, $0.14 \mathrm{mmol}$ ) and 3-(trifluoromethyl)-5-(2-pyridyl)pyrazole (34 mg, $0.16 \mathrm{mmol})$ were refluxed in anhydrous toluene $(10 \mathrm{~mL})$ for a period of $2 \mathrm{~h}$. The solvent was removed under vacuum and residue sublimed at $140{ }^{\circ} \mathrm{C}$ and 100 mTorr. Colorless crystals were obtained from a mixture of $\mathrm{CH}_{2} \mathrm{Cl}_{2}$ and diethyl ether $(58 \mathrm{mg}, 0.10 \mathrm{mmol}$, $68 \%)$.

Alternative Synthesis for 1. A finely pulverized mixture of [Os$\left.(\mathrm{CO})_{3}(\mathrm{tfa})_{2}\right](100 \mathrm{mg}, 0.20 \mathrm{mmol})$ and 3-(trifluoromethyl)-5-(2pyridyl)pyrazole (47 mg, $0.22 \mathrm{mmol}$ ) was loaded into a $10 \mathrm{~mL}$ Carius tube. The tube was sealed under vacuum and placed into an oven maintained at $185^{\circ} \mathrm{C}$ for $1 \mathrm{~h}$. After being allowed to cool to RT (room temperature), the tube was opened. The residue was triturated with distilled water and then sublimed at $140{ }^{\circ} \mathrm{C}$ and 100 mTorr. Further purification was carried out by recrystallization from a mixture of $\mathrm{CH}_{2} \mathrm{Cl}_{2}$ and diethyl ether, giving $85 \mathrm{mg}$ of colorless crystals $(0.14 \mathrm{mmol}, 71 \%)$.

Spectral data for 1: MS (EI, $\left.{ }^{192} \mathrm{Os}\right) \mathrm{m} / \mathrm{z}, 601\left(\mathrm{M}^{+}\right)$; IR $\left(\mathrm{CH}_{2-}\right.$ $\mathrm{Cl}_{2}$ ) v(CO), 2132 (vs), 2063 (vs), 2040 (vs) cm ${ }^{-1} ;{ }^{1} \mathrm{H}$ NMR (500 $\mathrm{MHz}$, acetone- $\left.d_{6}, 298 \mathrm{~K}\right) \delta 9.13\left(\mathrm{~d}, 1 \mathrm{H}, J_{\mathrm{HH}}=5.5 \mathrm{~Hz}, \mathrm{CH}\right), 8.34$ $\left(\mathrm{dt}, 1 \mathrm{H}, J_{\mathrm{HH}}=7.9,1.5 \mathrm{~Hz}, \mathrm{CH}\right), 8.22\left(\mathrm{~d}, 1 \mathrm{H}, J_{\mathrm{HH}}=7.5 \mathrm{~Hz}, \mathrm{CH}\right)$, $7.34\left(\mathrm{ddd}, 1 \mathrm{H}, J_{\mathrm{HH}}=6.0,5.5,1.5 \mathrm{~Hz}, \mathrm{CH}\right), 7.28(\mathrm{~s}, 1 \mathrm{H}, \mathrm{CH}) ;{ }^{13} \mathrm{C}$ NMR $\left(126 \mathrm{MHz}\right.$, acetone- $\left.d_{6}, 298 \mathrm{~K}\right) \delta 173.1(\mathrm{CO}), 169.6(\mathrm{CO})$, $167.5(\mathrm{CO}), 162.7\left(\mathrm{q},{ }^{2} J_{\mathrm{CF}}=37 \mathrm{~Hz}, C \mathrm{CF}_{3}\right), 155.4(\mathrm{CH}), 155.0$, $152.4,146.0\left(\mathrm{q},{ }^{2} J_{\mathrm{CF}}=37 \mathrm{~Hz},{C C_{F}}_{3}\right), 143.3(\mathrm{CH}), 125.4(\mathrm{CH})$, $122.9\left(\mathrm{q},{ }^{1} J_{\mathrm{CF}}=266 \mathrm{~Hz}, \mathrm{CF}_{3}\right), 122.2(\mathrm{CH}), 115.1\left(\mathrm{q},{ }^{1} J_{\mathrm{CF}}=287\right.$ $\left.\mathrm{Hz}, \mathrm{CF}_{3}\right), 104.0(\mathrm{CH}) ;{ }^{19} \mathrm{~F}$ NMR $\left(470 \mathrm{MHz}\right.$, acetone- $\left.d_{6}, 298 \mathrm{~K}\right) \delta$ -61.35 (s, 3F), -74.77 (s, 3F). Anal. Calcd for $\mathrm{C}_{14} \mathrm{H}_{5} \mathrm{~F}_{6} \mathrm{~N}_{3} \mathrm{O}_{5} \mathrm{Os}$ : C, 28.05; H, 0.84; N, 7.01. Found: C, 28.04; H, 1.16; N, 7.01.

Synthesis of $\left[\mathrm{Os}(\mathrm{CO})_{3}(\mathrm{Cl})(\mathrm{fppz})\right](2)$. A mixture of $\left[\mathrm{Os}(\mathrm{CO})_{3^{-}}\right.$ (tfa)(fppz)] (100 mg, $0.17 \mathrm{mmol})$ and $\mathrm{NaCl}(39 \mathrm{mg}, 0.67 \mathrm{mmol})$ in methanol $(25 \mathrm{~mL})$ was heated to reflux for $4 \mathrm{~h}$. After the reaction was completed, the solvent was evaporated in vacuum; the residue was washed with water and subjected to sublimation at $150{ }^{\circ} \mathrm{C}$ and 100 mTorr. Further purification was carried out by recrystallization from dichloromethane and diethyl ether, giving $78 \mathrm{mg}$ of white crystals $(0.15 \mathrm{mmol}, 90 \%)$. The respective bromide and iodide substituted derivatives $\left[\mathrm{Os}(\mathrm{CO})_{3}(\mathrm{Br})(\mathrm{fppz})\right](\mathbf{3})$ and $\left[\mathrm{Os}(\mathrm{CO})_{3}(\mathrm{I})\right.$ (fppz)] (4) were prepared by employing 4 equiv of halide reagents $\mathrm{NaBr}$ and $\mathrm{NaI}$, respectively.

Spectral data for 2: MS (EI, $\left.{ }^{192} \mathrm{Os}\right) \mathrm{m} / \mathrm{z}, 523\left(\mathrm{M}^{+}\right)$; IR $\left(\mathrm{CH}_{2^{-}}\right.$ $\mathrm{Cl}_{2}$ ) v(CO), 2128 (vs), 2058 (vs), 2032 (vs) cm ${ }^{-1} ;{ }^{1} \mathrm{H}$ NMR (500 $\mathrm{MHz}$, acetone- $\left.d_{6}, 298 \mathrm{~K}\right) \delta 9.09\left(\mathrm{~d}, 1 \mathrm{H}, J_{\mathrm{HH}}=6.0 \mathrm{~Hz}, \mathrm{CH}\right), 8.28$ $\left(\mathrm{dt}, 1 \mathrm{H}, J_{\mathrm{HH}}=7.9,1.0 \mathrm{~Hz}, \mathrm{CH}\right), 8.18\left(\mathrm{~d}, 1 \mathrm{H}, J_{\mathrm{HH}}=7.5 \mathrm{~Hz}, \mathrm{CH}\right)$, 7.57 (ddd, $\left.1 \mathrm{H}, J_{\mathrm{HH}}=8.0,6.0,2.0 \mathrm{~Hz}, \mathrm{CH}\right), 7.24(\mathrm{~s}, 1 \mathrm{H}, \mathrm{CH}) ;{ }^{13} \mathrm{C}$ NMR $\left(126 \mathrm{MHz}\right.$, acetone- $\left.d_{6}, 298 \mathrm{~K}\right) \delta 173.0(\mathrm{CO}), 170.1(\mathrm{CO})$, $165.5(\mathrm{CO}), 154.9,154.5(\mathrm{CH}), 151.8,145.7\left(\mathrm{q},{ }^{2} J_{\mathrm{CF}}=37 \mathrm{~Hz}\right.$, $\left.\mathrm{CCF}_{3}\right), 142.6(\mathrm{CH}), 125.4(\mathrm{CH}), 123.0\left(\mathrm{q},{ }^{1} J_{\mathrm{CF}}=266 \mathrm{~Hz}, \mathrm{CF}_{3}\right)$, $122.2(\mathrm{CH}), 104.0(\mathrm{CH}) ;{ }^{19} \mathrm{~F}$ NMR $\left(470 \mathrm{MHz}\right.$, acetone- $\left.d_{6}, 298 \mathrm{~K}\right)$ $\delta-61.17$ (s, 3F). Anal. Calcd for $\mathrm{C}_{12} \mathrm{H}_{5} \mathrm{ClF}_{3} \mathrm{~N}_{3} \mathrm{O}_{3} \mathrm{Os}$ : C, 27.62; H, 0.97; N, 8.05. Found: C, 27.58; H, 1.16; N, 7.84.

Spectral data for 3: MS (EI, $\left.{ }^{192} \mathrm{Os}\right) \mathrm{m} / \mathrm{z} 567\left(\mathrm{M}^{+}\right)$; IR $\left(\mathrm{CH}_{2^{-}}\right.$ $\mathrm{Cl}_{2}$ ) v(CO), 2127 (vs), 2058 (vs), 2033 (vs) $\mathrm{cm}^{-1}$; ${ }^{1} \mathrm{H}$ NMR (500 $\mathrm{MHz}$, acetone- $\left.d_{6}, 298 \mathrm{~K}\right) \delta 9.10\left(\mathrm{~d}, 1 \mathrm{H}, J_{\mathrm{HH}}=5.5 \mathrm{~Hz}, \mathrm{CH}\right), 8.28$ $\left(\mathrm{dt}, 1 \mathrm{H}, J_{\mathrm{HH}}=7.8,2.0 \mathrm{~Hz}, \mathrm{CH}\right), 8.19\left(\mathrm{~d}, 1 \mathrm{H}, J_{\mathrm{HH}}=8.0 \mathrm{~Hz}, \mathrm{CH}\right)$, $7.56\left(\mathrm{ddd}, 1 \mathrm{H}, J_{\mathrm{HH}}=7.0,6.0,1.0 \mathrm{~Hz}, \mathrm{CH}\right), 7.25(\mathrm{~s}, 1 \mathrm{H}, \mathrm{CH}) ;{ }^{13} \mathrm{C}$ NMR (126 MHz, acetone- $\left.d_{6}, 298 \mathrm{~K}\right) \delta 172.6(\mathrm{CO}), 169.9(\mathrm{CO})$, $164.5(\mathrm{CO}), 155.0,154.7(\mathrm{CH}), 151.8,145.8\left(\mathrm{q},{ }^{2} J_{\mathrm{CF}}=37 \mathrm{~Hz}\right.$, $\left.C \mathrm{CF}_{3}\right), 142.6(\mathrm{CH}), 125.4(\mathrm{CH}), 123.0\left(\mathrm{q},{ }^{1} J_{\mathrm{CF}}=268 \mathrm{~Hz}, \mathrm{CF}_{3}\right)$, $122.3(\mathrm{CH}), 104.0(\mathrm{CH}) ;{ }^{19} \mathrm{~F}$ NMR $\left(470 \mathrm{MHz}\right.$, acetone- $\left.d_{6}, 298 \mathrm{~K}\right)$ $\delta-61.16(\mathrm{~s}, 3 \mathrm{~F})$. Anal. Calcd for $\mathrm{C}_{12} \mathrm{H}_{5} \mathrm{BrF}_{3} \mathrm{~N}_{3} \mathrm{O}_{3} \mathrm{Os}$ : C, 25.45; H, 0.89; N, 7.42. Found: C, 25.46; H, 1.03; N, 7.21.

Spectral data for 4: MS (EI, $\left.{ }^{192} \mathrm{Os}\right) \mathrm{m} / \mathrm{z} 615\left(\mathrm{M}^{+}\right)$; IR $\left(\mathrm{CH}_{2^{-}}\right.$ $\mathrm{Cl}_{2}$ ) v(CO), 2123 (vs), 2054 (vs), 2032 (vs) cm ${ }^{-1} ;{ }^{1} \mathrm{H}$ NMR (500 $\mathrm{MHz}$, acetone- $\left.d_{6}, 298 \mathrm{~K}\right) \delta 9.13\left(\mathrm{~d}, J_{\mathrm{HH}}=5.6 \mathrm{~Hz}, 1 \mathrm{H}\right), 8.26(\mathrm{dt}$, $\left.J_{\mathrm{HH}}=8.0,1.0 \mathrm{~Hz}, 1 \mathrm{H}\right), 8.19\left(\mathrm{~d}, J_{\mathrm{HH}}=8.0 \mathrm{~Hz}, 1 \mathrm{H}\right), 7.54\left(\mathrm{ddd}, J_{\mathrm{HH}}\right.$ $=8.0,6.0,2.0 \mathrm{~Hz}, 1 \mathrm{H}), 7.27(\mathrm{~s}, 1 \mathrm{H}) ;{ }^{13} \mathrm{C} \mathrm{NMR}(126 \mathrm{MHz}$, acetone$\left.d_{6}, 298 \mathrm{~K}\right) \delta 172.1(\mathrm{CO}), 169.8(\mathrm{CO}), 162.9(\mathrm{CO}), 155.1(\mathrm{CH})$, $151.9(2 \mathrm{C}), 146.0\left(\mathrm{q},{ }^{2} J_{\mathrm{CF}}=37 \mathrm{~Hz}, \mathrm{CCF}_{3}\right), 142.4(\mathrm{CH}), 125.3(\mathrm{CH})$, $122.9\left(\mathrm{q},{ }^{1} J_{\mathrm{CF}}=267 \mathrm{~Hz}, \mathrm{CF}_{3}\right), 122.3(\mathrm{CH}), 104.1(\mathrm{CH}) ;{ }^{19} \mathrm{~F}$ NMR $\left(470 \mathrm{MHz}\right.$, acetone- $\left.d_{6}, 298 \mathrm{~K}\right) \delta-61.1(\mathrm{~s}, 3 \mathrm{~F})$. Anal. Calcd for $\mathrm{C}_{12} \mathrm{H}_{5} \mathrm{~F}_{3} \mathrm{IN}_{3} \mathrm{O}_{3} \mathrm{Os}$ : C, 23.50; $\mathrm{H}, 0.82 ; \mathrm{N}, 6.85$. Found: C, 23.68; $\mathrm{H}, 1.16$; N, 6.82 .

Synthesis of $\left[\mathrm{Os}(\mathrm{CO})_{3}(\mathbf{t f a})(\mathbf{f b t z})\right](5)$. Finely pulverized $\left[\mathrm{Os}_{2}-\right.$ $\left.(\mathrm{CO})_{6}(\mathrm{tfa})_{2}\right](100 \mathrm{mg}, 0.13 \mathrm{mmol})$ and 3-(trifluoromethyl)-5-(4-tertbutyl-2-pyridyl)-1,2,4-triazole $(77 \mathrm{mg}, 0.28 \mathrm{mmol})$ were mixed uniformly and loaded into a $10 \mathrm{~mL}$ Carius tube. The tube was sealed under vacuum and placed into an oven maintained at $185^{\circ} \mathrm{C}$ for 1 h. After the tube was cooled to room temperature, the material was subjected to sublimation at $150{ }^{\circ} \mathrm{C}$ and 100 mTorr. Further purification was carried out by recrystallization from dichloromethane and diethyl ether, giving $55 \mathrm{mg}$ of white crystals $(0.084$ mmol, $32 \%$ ).

Spectral data for 5: MS (EI, $\left.{ }^{192} \mathrm{Os}\right) \mathrm{m} / \mathrm{z}, 658\left(\mathrm{M}^{+}\right)$; IR $\left(\mathrm{CH}_{2^{-}}\right.$ $\mathrm{Cl}_{2}$ ) $v(\mathrm{CO}), 2134$ (vs), 2066 (vs), 2046 (vs) cm ${ }^{-1} ;{ }^{1} \mathrm{H}$ NMR (500 $\mathrm{MHz}$, acetone- $\left.d_{6}, 298 \mathrm{~K}\right) \delta 9.12\left(\mathrm{~d}, 1 \mathrm{H}, J_{\mathrm{HH}}=6.0 \mathrm{~Hz}\right), 8.26(\mathrm{~d}$, $\left.1 \mathrm{H}, J_{\mathrm{HH}}=2.5 \mathrm{~Hz}\right), 7.84\left(\mathrm{dd}, 1 \mathrm{H}, J_{\mathrm{HH}}=6.0,2.5 \mathrm{~Hz}\right), 1.48(\mathrm{~s}, 9 \mathrm{H})$; ${ }^{13} \mathrm{C}$ NMR (125.7 MHz, acetone- $\left.d_{6}, 298 \mathrm{~K}\right) \delta 172.1$ (CO), 169.0 (CO), $168.9(\mathrm{CO}), 166.4(\mathrm{CO}), 165.8,162.7\left(\mathrm{q},{ }^{2} J_{\mathrm{CF}}=38 \mathrm{~Hz}\right.$, $\left.C_{C F_{3}}\right), 157.2\left(\mathrm{q},{ }^{2} J_{\mathrm{CF}}=37 \mathrm{~Hz}, C \mathrm{CF}_{3}\right), 155.7(\mathrm{CH}), 149.9,125.1$ $(\mathrm{CH}), 121.0\left(\mathrm{q},{ }^{1} J_{\mathrm{CF}}=270 \mathrm{~Hz}, \mathrm{CF}_{3}\right), 120.4(\mathrm{CH}), 115.0\left(\mathrm{q},{ }^{1} J_{\mathrm{CF}}=\right.$ $\left.289 \mathrm{~Hz}, \mathrm{CF}_{3}\right), 36.7\left(\mathrm{CMe}_{3}\right), 30.2(3 \mathrm{C}) ;{ }^{19} \mathrm{~F} \mathrm{NMR}(470 \mathrm{MHz}$, acetone- $\left.d_{6}, 298 \mathrm{~K}\right) \delta-64.60(\mathrm{~s}, 3 \mathrm{~F}),-74.88(\mathrm{~s}, 3 \mathrm{~F})$. Anal. Calcd for $\mathrm{C}_{17} \mathrm{H}_{12} \mathrm{~F}_{6} \mathrm{~N}_{4} \mathrm{O}_{5} \mathrm{Os}$ : C, 31.10; $\mathrm{H}, 1.84 ; \mathrm{N}, 8.53$. Found: C, 31.14; $\mathrm{H}, 2.12 ; \mathrm{N}, 8.33$.

Synthesis of $\left[\mathrm{Os}(\mathrm{tfa})(\mathrm{fppz})\left(\mathrm{PPh}_{2} \mathrm{Me}\right)_{2}(\mathrm{CO})\right](6-8)$. An acetonitrile solution $(5 \mathrm{~mL})$ of freshly sublimed $\mathrm{Me}_{3} \mathrm{NO}(27 \mathrm{mg}, 0.365$ $\mathrm{mmol}$ ) was added dropwise into a stirred solution of $\mathbf{1}(200 \mathrm{mg}$, $0.332 \mathrm{mmol})$ in toluene $(25 \mathrm{~mL})$ over a period of $5 \mathrm{~min}$. This mixture was refluxed for $1 \mathrm{~h}, \mathrm{PPh}_{2} \mathrm{Me}(90 \mu \mathrm{L}, 0.498 \mathrm{mmol})$ was added, and the mixture was heated at reflux for another $12 \mathrm{~h}$. Finally, the solvent was removed under vacuum and the residue loaded onto a silica gel column and eluted with a 1:1 mixture of ethyl acetate and hexane, giving complexes 6 (105 mg, $0.111 \mathrm{mmol}$, $33 \%$ ), 7 (93 mg, $0.098 \mathrm{mmol}, 30 \%$ ), and 8 (13 mg, $0.013 \mathrm{mmol}$, $4 \%$ ) as yellow, pale yellow, and colorless powdery materials. Single crystals of all complexes were obtained by cooling a warm, saturated methanol solution to room temperature.

Spectral data for 6: MS (FAB, $\left.{ }^{192} \mathrm{Os}\right) \mathrm{m} / \mathrm{z}, 946\left(\mathrm{M}+1^{+}\right), 832$ $\left(\mathrm{M}^{+}-\mathrm{tfa}\right), 745\left(\mathrm{M}^{+}-\mathrm{PPh}_{2} \mathrm{Me}\right) ; \mathrm{IR}\left(\mathrm{CH}_{2} \mathrm{Cl}_{2}\right) v(\mathrm{CO}), 1955$ (vs) $\mathrm{cm}^{-1} ;{ }^{1} \mathrm{H}$ NMR $\left(500 \mathrm{MHz}\right.$, acetone- $\left.d_{6}, 298 \mathrm{~K}\right) \delta 8.39\left(\mathrm{~d}, 1 \mathrm{H}, J_{\mathrm{HH}}\right.$ $=5.5 \mathrm{~Hz}), 7.62\left(\mathrm{t}, 1 \mathrm{H}, J_{\mathrm{HH}}=7.5 \mathrm{~Hz}\right), 7.45-7.41(\mathrm{~m}, 4 \mathrm{H}), 7.35(\mathrm{t}$, 
$\left.2 \mathrm{H}, J_{\mathrm{HH}}=8.0 \mathrm{~Hz}\right), 7.25\left(\mathrm{t}, 6 \mathrm{H}, J_{\mathrm{HH}}=8.0 \mathrm{~Hz}\right), 7.17\left(\mathrm{~d}, 1 \mathrm{H}, J_{\mathrm{HH}}=\right.$ $7.5 \mathrm{~Hz}), 7.09-7.05$ (m, 5H), 6.95-6.91 (m, 4H), $6.56(\mathrm{~s}, 1 \mathrm{H}), 1.73$ $\left(\mathrm{t}, 6 \mathrm{H}, J_{\mathrm{HP}}=4.0 \mathrm{~Hz}\right) ;{ }^{19} \mathrm{~F}$ NMR $\left(470.3 \mathrm{MHz}\right.$, acetone- $\left.d_{6}, 298 \mathrm{~K}\right)$ $\delta-60.66(\mathrm{~s}, 3 \mathrm{~F}),-74.89(\mathrm{~s}, 3 \mathrm{~F}) ;{ }^{31} \mathrm{P}$ NMR $(202.3 \mathrm{MHz}$, acetone$\left.d_{6}, 298 \mathrm{~K}\right) \delta-10.81$ (s). Anal. Calcd for $\mathrm{C}_{38} \mathrm{H}_{31} \mathrm{~F}_{6} \mathrm{~N}_{3} \mathrm{O}_{3} \mathrm{OsP}_{2}$ : C, 48.36; H, 3.31; N, 4.45. Found: C, 48.53; H, 3.55; N, 4.70.

Spectral data for 7: MS (FAB, $\left.{ }^{192} \mathrm{Os}\right) \mathrm{m} / \mathrm{z}, 946\left(\mathrm{M}+1^{+}\right), 832$ $\left(\mathrm{M}^{+}-\mathrm{tfa}\right), 745\left(\mathrm{M}^{+}-\mathrm{PPh}_{2} \mathrm{Me}\right)$; IR $\left(\mathrm{CH}_{2} \mathrm{Cl}_{2}\right) v(\mathrm{CO}), 1937$ (vs) $\mathrm{cm}^{-1} .{ }^{1} \mathrm{H}$ NMR $\left(500 \mathrm{MHz}\right.$, acetone- $\left.d_{6}, 298 \mathrm{~K}\right) \delta 8.25\left(\mathrm{~d}, 1 \mathrm{H}, J_{\mathrm{HH}}\right.$ $=5.5 \mathrm{~Hz}), 7.34-7.30(\mathrm{~m}, 4 \mathrm{H}), 7.28-7.24(\mathrm{~m}, 5 \mathrm{H}), 7.17-7.05$ $(\mathrm{m}, 12 \mathrm{H}), 6.95\left(\mathrm{~d}, 1 \mathrm{H}, J_{\mathrm{HH}}=8.5 \mathrm{~Hz}\right), 6.69\left(\mathrm{dt}, 2 \mathrm{H}, J_{\mathrm{HH}}=7.5,1.5\right.$ $\mathrm{Hz}), 2.00\left(\mathrm{t}, 6 \mathrm{H}, J_{\mathrm{HP}}=4.0 \mathrm{~Hz}\right) ;{ }^{19} \mathrm{~F} \mathrm{NMR}(470.3 \mathrm{MHz}$, acetone$\left.d_{6}, 298 \mathrm{~K}\right) \delta-60.49$ (s, 3F), -74.47 (s, 3F); ${ }^{31} \mathrm{P} \mathrm{NMR}(202.3 \mathrm{MHz}$, acetone- $\left.d_{6}, 298 \mathrm{~K}\right) \delta-11.72(\mathrm{~s})$. Anal. Calcd for $\mathrm{C}_{38} \mathrm{H}_{31} \mathrm{~F}_{6} \mathrm{~N}_{3} \mathrm{O}_{3}$ $\mathrm{OsP}_{2}$ : C, 48.36; H, 3.31; N, 4.45. Found: C,48.32; H, 3.45; N, 4.69.

Spectral data for 8: MS (FAB, $\left.{ }^{192} \mathrm{Os}\right) \mathrm{m} / z, 945\left(\mathrm{M}^{+}\right), 832\left(\mathrm{M}^{+}\right.$ - tfa); IR $\left(\mathrm{CH}_{2} \mathrm{Cl}_{2}\right) v(\mathrm{CO}), 1948$ (vs) $\mathrm{cm}^{-1} ;{ }^{1} \mathrm{H} \mathrm{NMR}(500 \mathrm{MHz}$, acetone- $\left.d_{6}, 298 \mathrm{~K}\right) \delta 8.05\left(\mathrm{~d}, 1 \mathrm{H}, J_{\mathrm{HH}}=6.5 \mathrm{~Hz}\right), 7.95-7.91(\mathrm{~m}$, $3 \mathrm{H}), 7.82\left(\mathrm{dt}, 1 \mathrm{H}, J_{\mathrm{HH}}=7.5,1.5 \mathrm{~Hz}\right), 7.62-7.55(\mathrm{~m}, 3 \mathrm{H}), 7.48$ $\left(\mathrm{dt}, 2 \mathrm{H}, J_{\mathrm{HH}}=7.3,2.5 \mathrm{~Hz}\right), 7.39-7.34(\mathrm{~m}, 3 \mathrm{H}), 7.28(\mathrm{~s}, 1 \mathrm{H}), 7.24$ $\left(\mathrm{dt}, 1 \mathrm{H}, J_{\mathrm{HH}}=7.5,1.5 \mathrm{~Hz}\right), 7.18-7.14(\mathrm{~m}, 5 \mathrm{H}), 7.06\left(\mathrm{dt}, 2 \mathrm{H}, J_{\mathrm{HH}}\right.$ $=7.5,2.5 \mathrm{~Hz}), 6.82-6.77(\mathrm{~m}, 3 \mathrm{H}), 2.39\left(\mathrm{~d}, 3 \mathrm{H}, J_{\mathrm{HP}}=10.5 \mathrm{~Hz}\right)$, $1.49\left(\mathrm{~d}, 3 \mathrm{H}, J_{\mathrm{HP}}=9.5 \mathrm{~Hz}\right) ;{ }^{19} \mathrm{~F}$ NMR $\left(470.3 \mathrm{MHz}\right.$, acetone- $d_{6}$, $298 \mathrm{~K}) \delta-60.90$ (s, 3F), -75.00 (s, 3F); ${ }^{31} \mathrm{P}$ NMR $(202.3 \mathrm{MHz}$, acetone- $\left.d_{6}, 298 \mathrm{~K}\right) \delta-15.15\left(\mathrm{~d}, J_{\mathrm{PP}}=11.1 \mathrm{~Hz}\right),-15.97\left(\mathrm{~d}, J_{\mathrm{PP}}=\right.$ $11.1 \mathrm{~Hz})$.

X-ray Crystallography. Single-crystal X-ray diffraction data was measured on a Bruker SMART CCD diffractometer $\left(2 \theta_{\max }\right.$ $\leq 55.0^{\circ}, \omega$ scan mode) equipped with a graphite monochromator. The data collection was executed using the SMART program. Cell refinement and data reduction were accomplished using the SAINT program. The structures were solved using the SHELXTL/PC package and refined using full-matrix least squares. An empirical absorption correction was applied with the SADABS routine (part of the SHELXTL program). The structure was solved by direct methods using the SHELXTL suite of programs. All non-hydrogen atoms were refined anisotropically by full-matrix least-squares on $F^{2}$. Hydrogen atoms were placed in calculated positions and allowed to ride on the parent carbon atoms.

Selected crystal data for 1: $\mathrm{C}_{14} \mathrm{H}_{5} \mathrm{~F}_{6} \mathrm{~N}_{3} \mathrm{O}_{5} \mathrm{Os}, M=599.41$; triclinic, space group $P \overline{1} ; a=9.9557(1), b=12.0647(2), c=$ 15.7194(3) $\AA ; \alpha=85.4865(10), \beta=74.0517(11), \gamma=71.1197$ $(11)^{\circ} ; V=1717.64(5) \AA^{3} ; T=150(2) \mathrm{K} ; Z=4 ; \mu($ Mo K $\alpha)=$ $7.521 \mathrm{~mm}^{-1} ; 24159$ reflections measured with 7880 unique reflections $\left(\mathrm{R}_{\text {int }}=0.0606\right)$; final $\mathrm{wR}_{2}($ all data $)=0.0844, \mathrm{R}_{1}[I>$ $2 \sigma(I)]=0.0326$.

Selected crystal data for 6: $\mathrm{C}_{38} \mathrm{H}_{31} \mathrm{~F}_{6} \mathrm{~N}_{3} \mathrm{O}_{3} \mathrm{OsP}_{2}, M=943.80$; monoclinic, space group $P 2_{1} / c ; a=12.5949(8), b=18.2927(11)$, $c=16.1595(10) \AA ; \beta=101.664(2)^{\circ} ; V=3646.2(4) \AA^{3} ; T=150$ (2) $\mathrm{K} ; Z=4 ; \mu($ Mo $\mathrm{K} \alpha)=3.658 \mathrm{~mm}^{-1} ; 21772$ reflections measured with 6406 unique reflections $\left(\mathrm{R}_{\text {int }}=0.0841\right)$; final $w \mathrm{R}_{2}$ (all data) $=0.1730, \mathrm{R}_{1}[I>2 \sigma(I)]=0.0930$.

Selected crystal data for 7: $\mathrm{C}_{38} \mathrm{H}_{31} \mathrm{~F}_{6} \mathrm{~N}_{3} \mathrm{O}_{3} \mathrm{OsP}_{2}, M=943.80$; triclinic, space group $P \overline{1} ; a=9.4227(7), b=11.2810(8), c=$ 17.8178(13) $\AA ; \alpha=77.385(2), \beta=84.591(2), \gamma=75.979(2)^{\circ} ; V$ $=1791.5(2) \AA^{3} ; T=150(2) \mathrm{K} ; Z=2 ; \mu(\mathrm{Mo} \mathrm{K} \alpha)=3.722 \mathrm{~mm}^{-1}$; 23330 reflections measured with 8215 unique reflections $\left(\mathrm{R}_{\text {int }}=\right.$ 0.0326 ); final $\mathrm{wR}_{2}$ (all data) $=0.0637, \mathrm{R}_{1}[I>2 \sigma(I)]=0.0248$.

Selected crystal data for 8: $\mathrm{C}_{38} \mathrm{H}_{31} \mathrm{~F}_{6} \mathrm{~N}_{3} \mathrm{O}_{3} \mathrm{OsP}_{2}, M=943.80$; triclinic, space group $P \overline{1} ; a=10.2656(7), b=12.4189(8), c=$ 14.7477(9) $\AA$; $\alpha=92.357(1), \beta=92.615(1), \gamma=109.768(1)^{\circ} ; V$ $=1764.3(2) \AA^{3} ; T=150(2) \mathrm{K} ; Z=2 ; \mu($ Mo K $\alpha)=3.780 \mathrm{~mm}^{-1}$;
19008 reflections measured with 6225 unique reflections $\left(\mathrm{R}_{\mathrm{int}}=\right.$ 0.0867 ); final $\mathrm{wR}_{2}$ (all data) $=0.1250, \mathrm{R}_{1}[I>2 \sigma(I)]=0.0716$.

Computational Methodology. Calculations on the electronic ground states of complexes $\mathbf{1 - 8}$ were carried out using B3LYP density functional theory. ${ }^{12-13} \mathrm{~A}$ "double- $\zeta$ " quality basis set consisting of Hay and Wadt's effective core potentials (LANL2DZ) ${ }^{12}$ was employed for Os atoms, and a $6-31 \mathrm{G}^{*}$ basis, ${ }^{14}$ for $\mathrm{H}, \mathrm{C}, \mathrm{N}$, and $\mathrm{F}$ atoms. A relativistic effective core potential (ECP) replaced the inner core electrons of the Os(II) element, leaving the outer core $\left(5 s^{2} 5 p^{6}\right)$ electrons and the $5 d^{6}$ valence electrons. Timedependent DFT (TDDFT) calculations using the B3LYP functional were then performed on the basis of the structural optimized geometries. ${ }^{15}$ Typically, the lowest 10 triplet and 10 singlet roots of the nonhermitian eigenvalue equations were obtained to determine the vertical excitation energies. Oscillator strengths were deduced from the dipole transition matrix elements (for singlet states only). The ground-state B3LYP and excited-state TDDFT calculations were carried out using Gaussian03. ${ }^{16}$ Compositions of molecular orbitals in terms of the constituent chemical fragments were calculated using the AOMix program. ${ }^{17}$ For the characterization of the HOMO- $x \rightarrow$ LUMO $+y$ transitions as partial charge transfer (CT) transitions, the following definition of the CT character has been used:

$$
\mathrm{CT}(\mathrm{M})=\%(\mathrm{M}) \mathrm{HOMO}-x-\%(\mathrm{M}) \mathrm{LUMO}+y
$$

Here $\%(\mathrm{M}) \mathrm{HOMO}-x$ and $\%(\mathrm{M}) \mathrm{LUMO}+y$ are electronic densities on the metal in HOMO- $x$ and LUMO $+y$. If the excited state, e.g., $S_{1}$ or $T_{1}$, is formed by more than one one-electron excitation, then the metal CT character of this excited-state is expressed as a sum of CT characters of each participating excitation, $i \rightarrow j$ :

$$
\mathrm{CT}_{I}(\mathrm{M})=\sum_{i, \mathrm{a}}\left[\mathrm{C}_{I}(i \rightarrow j)\right]^{2}\left(\%(\mathrm{M})_{i}-\%(\mathrm{M})_{j}\right)
$$

Here $\mathrm{C}_{I}(i \rightarrow j)$ are the appropriate coefficients of the Ith eigenvector of the CT matrix. Accordingly, one can very effectively use the

(12) (a) Lee, C.; Yang, W.; Parr, R. G. Phys. Rev. B 1988, 37, 785. (b) Becke, A. D. J. Chem. Phys. 1993, 98, 5648.

(13) (a) Hay, P. J.; Wadt, W. R. J. Chem. Phys. 1985, 82, 270. (b) Wadt, W. R.; Hay, P. J. J. Chem. Phys. 1985, 82, 284. (c) Hay, P. J.; Wadt, W. R. J. Chem. Phys. 1985, 82, 299.

(14) Hariharan, P. C.; Pople, J. A. Mol. Phys. 1974, 27, 209.

(15) (a) Jamorski, C.; Casida, M. E.; Salahub, D. R. J. Chem. Phys. 1996, 104, 5134. (b) Petersilka, M.; Grossmann, U. J.; Gross, E. K. U. Phys. Rev. Lett. 1996, 76, 1212. (c) Bauernschmitt, R.; Ahlrichs, R.; Hennrich, F. H.; Kappes, M. M. J. Am. Chem. Soc. 1998, 120, 5052 (d) Casida, M. E. J. Chem. Phys. 1998, 108, 4439. (e) Stratmann, R. E.; Scuseria, G. E.; Frisch, M. J. J. Chem. Phys. 1998, 109, 8218.

(16) Frisch, M. J.; Trucks, G. W.; Schlegel, H. B.; Scuseria, G. E.; Robb, M. A.; Cheeseman, J. R.; Montgomery, J. A., Jr.; Vreven, T.; Kudin, K. N.; Burant, J. C.; Millam, J. M.; Iyengar, S. S.; Tomasi, J.; Barone, V.; Mennucci, B.; Cossi, M.; Scalmani, G.; Rega, N.; Petersson, G. A.; Nakatsuji, H.; Hada, M.; Ehara, M.; Toyota, K.; Fukuda, R.; Hasegawa, J.; Ishida, M.; Nakajima, T.; Honda, Y.; Kitao, O.; Nakai, H.; Klene, M.; Li, X.; Knox, J. E.; Hratchian, H. P.; Cross, J. B.; Bakken, V.; Adamo, C.; Jaramillo, J.; Gomperts, R.; Stratmann, R. E.; Yazyev, O.; Austin, A. J.; Cammi, R.; Pomelli, C.; Ochterski, J. W.; Ayala, P. Y.; Morokuma, K.; Voth, G. A.; Salvador, P.; Dannenberg, J. J.; Zakrzewski, V. G.; Dapprich, S.; Daniels, A. D.; Strain, M. C.; Farkas, O.; Malick, D. K.; Rabuck, A. D.; Raghavachari, K.; Foresman, J. B.; Ortiz, J. V.; Cui, Q.; Baboul, A. G.; Clifford, S.; Cioslowski, J.; Stefanov, B. B.; Liu, G.; Liashenko, A.; Piskorz, P.; Komaromi, I.; Martin, R. L.; Fox, D. J.; Keith, T.; Al-Laham, M. A.; Peng, C. Y.; Nanayakkara, A.; Challacombe, M.; Gill, P. M. W.; Johnson, B.; Chen, W.; Wong, M. W.; Gonzalez, C.; Pople, J. A. Gaussian 03, revision C.02; Gaussian, Inc.: Wallingford, CT, 2004

(17) (a) Gorelsky, S. I. AOMix: Program, for Molecular, Orbital, Analysis; http://www.sg-chem.net/; University of Ottawa: Ottawa, Canada, 2007. (b) Gorelsky, S. I.; Lever, A. B. P. J. Organomet. Chem. 2001, 635, 187-196. 
MO compositions in terms of fragment orbital contributions to probe the nature of electronic transitions. More details about the basis of this method could be found in the manual of the AOMix program.

\section{Results and Discussion}

Preparation and Characterization. The blue-emitting complex $\left[\mathrm{Os}(\mathrm{CO})_{3}(\mathrm{tfa})(\mathrm{fppz})\right](\mathbf{1})$ was prepared by treatment of $\mathrm{Os}(\mathrm{II})$ reagent $\left[\mathrm{Os}(\mathrm{CO})_{3}(\mathrm{tfa})_{2}\right]\left(\mathrm{tfa}={\text { trifluoroacetate })^{18}}^{18}\right.$ and 3-(trifluoromethyl)-5-(2-pyridyl)pyrazole (fppz)H in refluxing toluene or using the so-called solid-state pyrolysis technique. ${ }^{19}$ Both methods were initially utilized for the preparation of emissive Os(II) complexes. ${ }^{20}$ After isolation and purification of $\mathbf{1}$, its IR $v(\mathrm{CO})$ data showed three $\mathrm{CO}$ stretching bands at 2132, 2063, and $2040 \mathrm{~cm}^{-1}$ in $\mathrm{CH}_{2} \mathrm{Cl}_{2}$, which are characteristic for a facial- $\left[\mathrm{Os}(\mathrm{CO})_{3}\right]$ unit. This complex was then characterized with single-crystal X-ray diffraction analyses. As depicted in Figure 1, the structure consists of a central Os(II) atom chelated by an fppz ligand, together with the attachment of one terminal tfa anion and three $\mathrm{CO}$ ligands. Its coordination arrangement is akin to that of the diketonate complexes $\left[\mathrm{Os}(\mathrm{CO})_{3}(\mathrm{tfa})(\mathrm{hfac})\right]$, hfac $=$ hexafluoroacetylacetonate ${ }^{21}$ and the 8-quinolinolate derivative $\left[\mathrm{Os}(\mathrm{CO})_{3}(\mathrm{tfa})\left(\mathrm{O}^{\wedge} \mathrm{N}\right)\right], \mathrm{O}^{\wedge} \mathrm{N}=$ quinolinolate; the latter even shows the room-temperature dual fluorescence and phosphorescence arising from inefficient singlet-triplet intersystem crossing. ${ }^{22}$

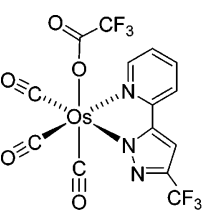

(1)

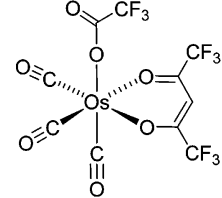

$\left[\mathrm{Os}(\mathrm{CO})_{3}(\mathrm{ffa})(\mathrm{hfac})\right]$

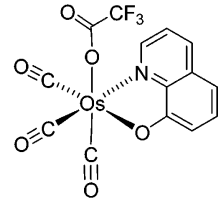

$\left[\mathrm{Os}(\mathrm{CO})_{3}(\mathrm{ffa})\left(\mathrm{N}^{\wedge} \mathrm{O}\right)\right]$
Other halide-substituted derivatives $\mathbf{2}-\mathbf{4}$ were synthesized via an anion exchange reaction, which involved direct treatment of parent tfa complex 1 with the alkali metal salts $\mathrm{NaCl}, \mathrm{NaBr}$, and $\mathrm{NaI}$ in refluxing methanol solution. These complexes were then identified using spectroscopic methods, for which the distinct IR $v(\mathrm{CO})$ patterns are essentially identical with that of $\mathbf{1}$ and show a slightly decreasing in stretching frequency with a sequence of $\mathrm{Cl} \geq \mathrm{Br} \geq \mathrm{I}$, which is consistent with the trend of $\pi$-donor strength and/or the electronegativity of the halides. As for the photophysical characteristics, the lowest lying electronic transition for 1-4 may be largely attributed to the unique fppz ligand (vide infra). Accordingly, as for further strategic design, the pyridyl

(18) (a) Deeming, A. J.; Meah, M. N.; Randle, N. P.; Hardcastle, K. I. J. Chem. Soc., Dalton Trans. 1989, 2211. (b) Deeming, A. J.; Randle, N. P.; Bates, P. A.; Hursthouse, M. B. J. Chem. Soc. Dalton Trans. 1988, 2753.

(19) Gong, J.-H.; Hwang, D.-K.; Tsay, C.-W.; Chi, Y.; Peng, S.-M.; Lee, G.-H. Organometallics 1994, 13, 1720.

(20) Yu, J.-K.; Cheng, Y.-M.; Hu, Y.-H.; Chou, P.-T.; Chen, Y.-L.; Lee, S.-W.; Chi, Y. J. Phys. Chem. B 2004, 108, 19908.

(21) (a) Chen, Y.-L.; Li, S.-W.; Chi, Y.; Cheng, Y.-M.; Pu, S.-C.; Yeh, Y.-S.; Chou, P.-T. Chem. Phys. Chem. 2005, 6, 2012. (b) Chen, Y.L.; Sinha, C.; Chen, I.-C.; Liu, K.-L.; Chi, Y.; Yu, J.-K.; Chou, P.-T.; Lu, T.-H. Chem. Commun. 2003, 3046.

(22) Cheng, Y.-M.; Yeh, Y.-S.; Ho, M.-L.; Chou, P.-T.; Chen, P.-S.; Chi, Y. Inorg. Chem. 2005, 44, 4594. pyrazolate chelate in $\mathbf{1 - 4}$ is replaced by the tert-butylsubstituted pyridyl triazolate, forming derivative 5. In comparison to $\mathbf{1 - 4}$, we expect a profound increase on the associated emission energy gap due to its increase (decrease) of the electronic density in the pyridyl and the triazolate moieties, respectively.

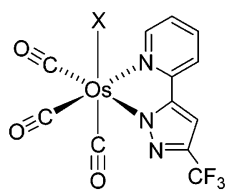

(2) $\mathrm{X}=\mathrm{Cl}$; (3) $\mathrm{X}=\mathrm{Br} ;(4) \mathrm{X}=1$

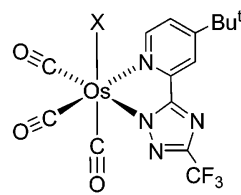

(5) $\mathrm{X}=\mathrm{CF}_{3} \mathrm{CO}_{2}$
Last, we also conducted the phosphine substitution reaction with an aim to probe the influence of ancillary ligands on their structural and photophysical properties. The results of this attempt led to the successful isolation of three $\mathrm{PPh}_{2} \mathrm{Me}$ substituted complexes $\mathbf{6}-\mathbf{8}$ in 30,33 , and $4 \%$ yields, respectively. Spectroscopic investigation revealed that complexes 6-8 were isomeric, as all possess one pyridyl pyrazolate chelate, one carbonyl, and one terminal tfa ligand attached to the central Os(II) atom, together with two $\mathrm{PPh}_{2}$ Me ligands (vide infra). It is also noted that although the crystalline samples are indefinitely stable in air at room temperature, they turned unstable upon being dissolved in both chlorinated solvents and even nonchlorinated solvent such as acetone. A color change from light yellow to dark green was observed within $24 \mathrm{~h}$ for the solutions prepared for NMR analyses, showing high chemical reactivity that is in sharp contrast to that of the hydride analogues. ${ }^{23}$

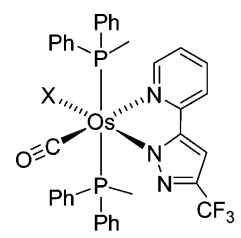

(6) $\mathrm{X}=\mathrm{CF}_{3} \mathrm{CO}_{2}$

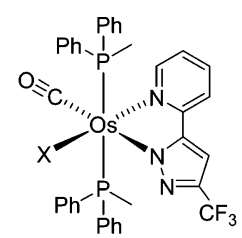

(7) $\mathrm{X}=\mathrm{CF}_{3} \mathrm{CO}_{2}$

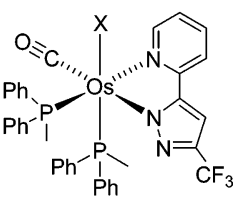

(8) $\mathrm{X}=\mathrm{CF}_{3} \mathrm{CO}_{2}$
Complexes 6 and 7 were also characterized through crystal structural analyses. Figures 2 and 3 reveal their molecular structures. The $\mathrm{PPh}_{2} \mathrm{Me}$ ligands in both complexes adopt a trans-configuration, with $\mathrm{Os}-\mathrm{P}$ distances in the range of 2.367-2.378 $\AA$, which are comparable to that of the redemitting Os(II) complex [Os(fppz $\left.)_{2}\left(\mathrm{PPh}_{2} \mathrm{Me}\right)_{2}\right](2.362 \AA)^{24}$ and the corresponding porphinato $(2.369 \AA)$ and benzotriazolato analogues $(2.353 \AA) .{ }^{25,26}$ Moreover, it is notable that the pyridyl group in $\mathbf{6}$ is located trans to the strong $\pi$-accepting $\mathrm{CO}$ ligand. It exhibits a lengthened Os $-\mathrm{N}(\mathrm{py})$ distance in $6(\mathrm{Os}-\mathrm{N}(1)=2.149 \AA)$, compared with that of

(23) Hsu, F.-C.; Tung, Y.-L.; Chi, Y.; Hsu, C.-C.; Cheng, Y.-M.; Ho, M.L.; Chou, P.-T.; Peng, S.-M.; Carty, A. J. Inorg. Chem. 2006, 45, 10188.

(24) Tung, Y.-L.; Wu, P.-C.; Liu, C.-S.; Chi, Y.; Yu, J.-K.; Hu, Y.-H.; Chou, P.-T.; Peng, S.-M.; Lee, G.-H.; Tao, Y.; Carty, A. J.; Shu, C.F.; Wu, F.-I. Organometallics 2004, 23, 3745

(25) Xie, J.; Huang, J.-S.; Zhu, N.; Zhou, Z.-Y.; Che, C.-M. Chem.-Eur. J. 2005, 11, 2405.

(26) Olby, B. G.; Robinson, S. D.; Hursthouse, M. B.; Short, R. L. J. Chem. Soc., Dalton Trans. 1990, 621. 


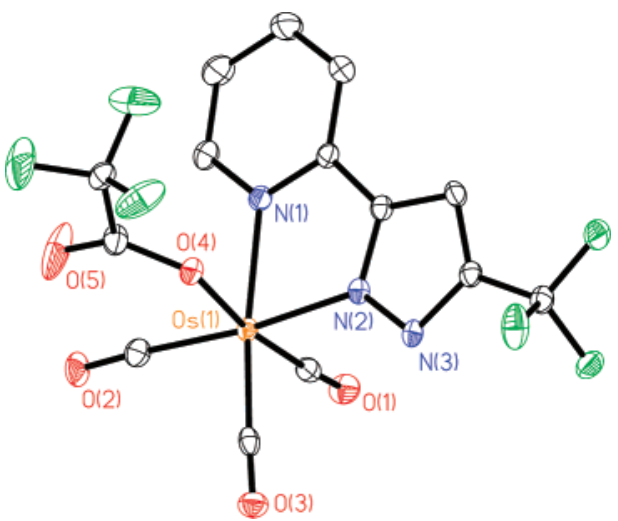

Figure 1. ORTEP drawing of complex 1 with thermal ellipsoids shown at the $30 \%$ probability level. Selected distances: Os(1) $-\mathrm{C}(1)=1.906(5)$, $\mathrm{Os}(1)-\mathrm{C}(2)=1.961(5), \mathrm{Os}(1)-\mathrm{C}(3)=1.938(5), \mathrm{Os}(1)-\mathrm{O}(4)=2.098$ (3), $\operatorname{Os}(1)-\mathrm{N}(1)=2.139(4)$, and $\operatorname{Os}(1)-\mathrm{N}(2)=2.061(4) \AA$.

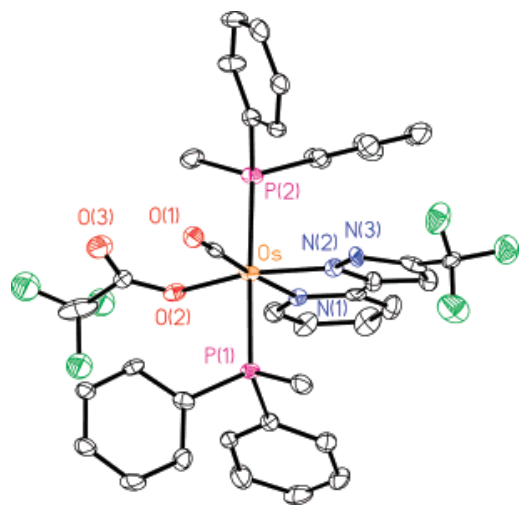

Figure 2. ORTEP drawing of complex 6 with thermal ellipsoids shown at the $30 \%$ probability level. Selected distances: Os $-\mathrm{P}(1)=2.367(3)$, Os$\mathrm{P}(2)=2.378(4), \mathrm{Os}-\mathrm{N}(1)=2.149(10), \mathrm{Os}-\mathrm{N}(2)=2.035(11), \mathrm{Os}-\mathrm{O}(2)$ $=2.112(10)$, and $\mathrm{Os}-\mathrm{C}(1)=1.832(15) \AA$.

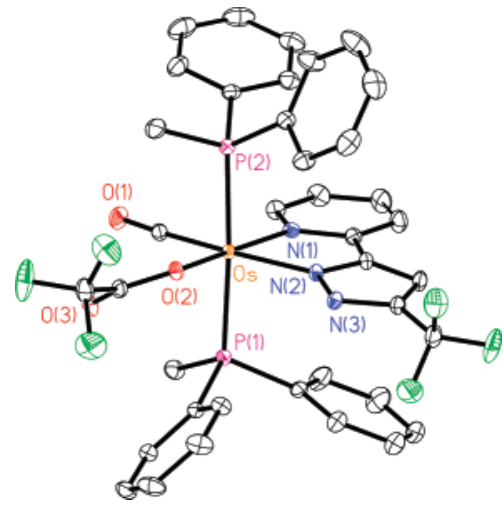

Figure 3. ORTEP drawing of complex 7 with thermal ellipsoids shown at the $30 \%$ probability level. Selected distances: Os $-\mathrm{P}(1)=2.3784(8)$, $\mathrm{Os}-\mathrm{P}(2)=2.3752(8), \mathrm{Os}-\mathrm{N}(1)=2.079(3), \mathrm{Os}-\mathrm{N}(2)=2.118(3)$, Os$\mathrm{C}(1)=1.845(3)$, and $\mathrm{Os}-\mathrm{O}(2)=2.120(2) \AA$.

its geometric isomer $7(\mathrm{Os}-\mathrm{N}(1)=2.079 \AA)$, for which the pyridyl unit is located trans to the somewhat $\sigma$-donating tfa ligand. The reverse alternation of the $\mathrm{Os}-\mathrm{N}$ bond distances is also detected for the pyrazolate segments, i.e., $2.035 \AA$ in 6 versus $2.118 \AA$ in 7 , indicating that the tfa ligand is a poor trans-directing ligand compared with the $\mathrm{CO}$ ligand within this class of complexes.

Finally, the structure of the third, nonemissive counterpart $\mathbf{8}$ was also subjected to the single-crystal X-ray structural

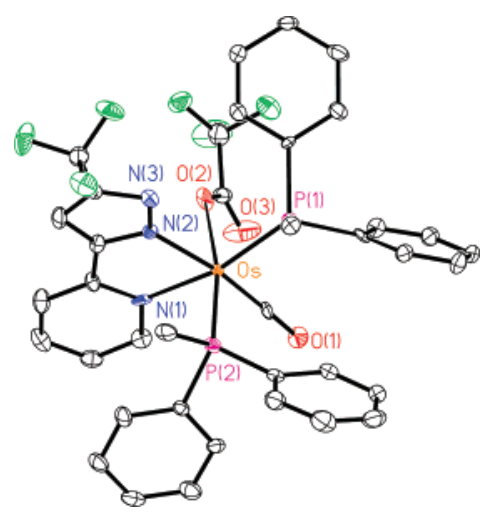

Figure 4. ORTEP drawing of complex 8 with thermal ellipsoids shown at the $30 \%$ probability level. Selected distances: $\mathrm{Os}-\mathrm{P}(1)=2.336(3)$, Os$\mathrm{P}(2)=2.310(3)$, Os $-\mathrm{N}(1)=2.141(9), \mathrm{Os}-\mathrm{N}(2)=2.109(8)$, Os $-\mathrm{C}(1)=$ $1.839(12)$, and $\mathrm{Os}-\mathrm{O}(2)=2.118(8) \AA$.

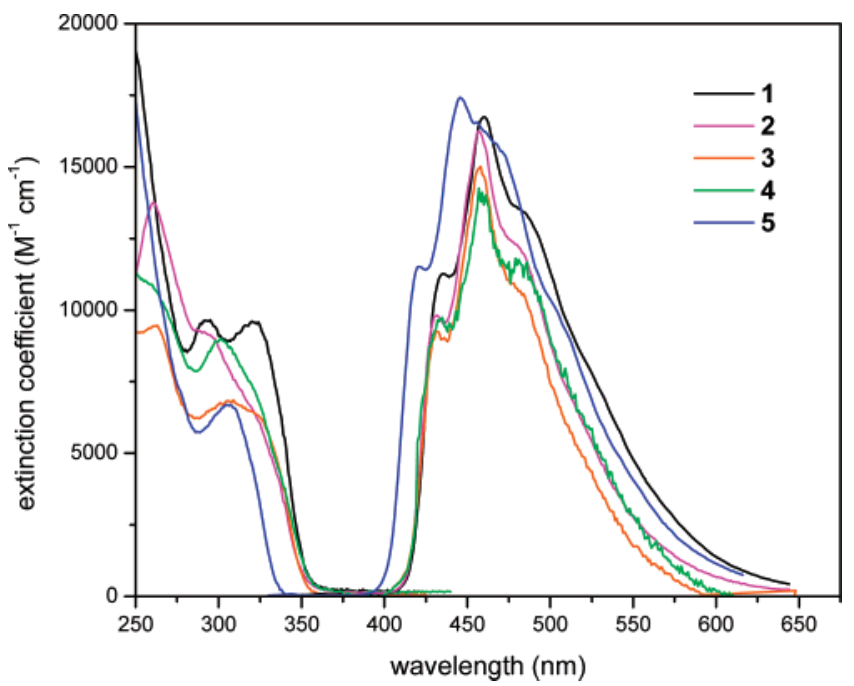

Figure 5. UV-vis absorption and emission spectra of Os(II) complexes 1-5 in room-temperature acetonitrile. Note that the normalized emission spectra were acquired under the degassed condition.

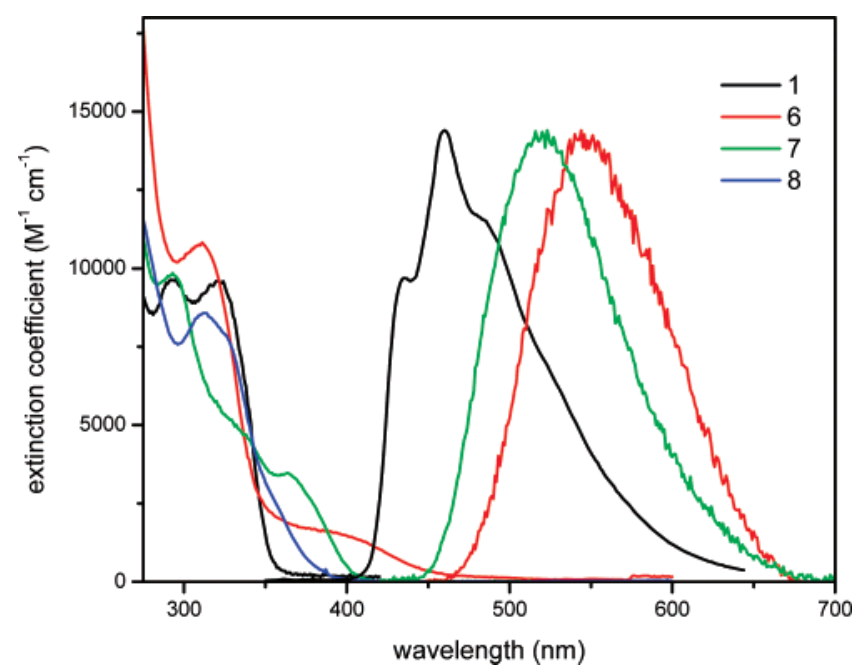

Figure 6. UV-vis absorption and emission spectra of Os(II) complexes $\mathbf{1}$ and $\mathbf{6}-\mathbf{8}$ in room-temperature acetonitrile. Note that the normalized emission spectra were acquired under the degassed condition.

determination. As shown in Figure 4, this complex depicts a distinctive configuration with the phosphine ligands located at the cis-disposition and showing notably stronger Os $-\mathrm{P}$ 
Cheng et al.

Table 1. Selected Photophysical Properties of Os(II) Complexes 1-8 in Acetonitrile at RT

\begin{tabular}{clcccc}
\hline compd & \multicolumn{1}{c}{ UV/vis data/nm $\left(10^{-3} \epsilon\right)$} & PL $\lambda_{\max }(\mathrm{nm})$ & QY & $\tau_{\text {obs }}(\mu \mathrm{s})$ & $k_{\mathrm{r}}$ \\
\hline $\mathbf{1}$ & $247(19), 292(9.6), 320(9.6)$ & 460 & 0.26 & 143 & $1.8 \times 10^{3}$ \\
$\mathbf{2}$ & $260(14), 291(8.8), 316(6.9)$ & 457 & 0.03 & 25.6 & $1.2 \times 10^{3}$ \\
$\mathbf{3}$ & $263(9.6), 306(6.9), 318(6.5)$ & 458 & 0.003 & 2.07 & $1.5 \times 10^{3}$ \\
$\mathbf{4}$ & $260(11), 301(9.0)$ & 457 & $<10^{-4}$ & 0.3 & $\sim 3.0 \times 10^{3}$ \\
$\mathbf{5}$ & $307(6.7)$ & 446 & 0.27 & 46.2 & $5.8 \times 10^{3}$ \\
$\mathbf{6}$ & $254(27), 270(21), 312(11), 398(1.5)$ & 550 & 0.002 & 0.024 & $8.3 \times 10^{5}$ \\
$\mathbf{7}$ & $270(18), 292(14), 330(6.7), 366(3.4)$ & 520 & 0.003 & 0.035 & $8.6 \times 10^{6}$ \\
$\mathbf{8}$ & $273(13), 309(8.5), 328(7.3), 360(3.3, \mathrm{sh})$ & & & & $4.2 \times 10^{7}$ \\
& & & &
\end{tabular}

interactions, Os $-\mathrm{P}(1)=2.336(3)$, and $\mathrm{Os}-\mathrm{P}(2)=2.310(3)$ $\AA$, due to the absence of trans phosphine-to-phosphine linear alignment. Moreover, the variation of $\mathrm{Os}-\mathrm{N}$ distances in $\mathbf{8}$ was found to be comparable with the respective Os $-\mathrm{N}$ lengths observed in both complexes $\mathbf{6}$ and $\mathbf{7}$, showing similar trans-influences exerted on the fppz ligand.

Photophysical Properties. The absorption and luminescence spectra recorded for $\mathbf{1 - 5}$ in acetonitrile are depicted in Figure 5, while pertinent data are listed in Table 1. Several remarks can be pointed out from the corresponding spectroscopic and dynamic measurements. As shown in the absorption spectra, complexes $\mathbf{1 - 4}$ bearing different axial anions, i.e., either tfa or halide ions, share spectral similarities in the lower lying electronic transitions. For example, they all exhibit the $S_{0} \rightarrow S_{1}$ transition band in the UV region of ca. 300-350 nm with high absorptivity of $\epsilon \sim 1.0 \times 10^{4}$ $\mathrm{M}^{-1} \mathrm{~cm}^{-1}$ at their peak maxima of $\sim 320 \mathrm{~nm}$. The results can be rationalized by the $S_{0} \rightarrow S_{1}$ transition for $\mathbf{1}-\mathbf{4}$ being ascribed to the dominant ligand-based $\pi \pi^{*}$ transition from pyrazolate to the pyridyl moiety of the fppz chelate (not shown here). ${ }^{6 a}$ Another support of this viewpoint is given by the comparative study between $\mathbf{1 - 4}$ and $\mathbf{5}$. Adding a tertbutyl group on the pyridyl moiety and substitution of pyrazolate site by triazolate to $\mathbf{1}$, forming complex $\mathbf{5}$, gives rise to an $\mathrm{S}_{0} \rightarrow \mathrm{S}_{1}$ transition at $305 \mathrm{~nm}$, which is significantly blue-shifted from that $(\sim 320 \mathrm{~nm})$ of $\mathbf{1}-\mathbf{4}$. The results are best rationalized by the $S_{0} \rightarrow S_{1}$ transition for $\mathbf{1}-\mathbf{5}$ being largely attributed to azolate (HOMO) pyridyl (LUMO) $\pi \pi^{*}$ transition, such that a significant increase of the associated $\mathrm{S}_{0} \rightarrow \mathrm{S}_{1}$ energy gap is expected for $\mathbf{5}$ due to its profound decrease (increase) of the electron density in the HOMO (LUMO) level. Further direct support will be elucidated in the section on computational approaches.

In yet another approach, despite their structural similarities, three isomers, $\mathbf{6}-\mathbf{8}$, which are formed by replacing two terminal $\mathrm{CO}$ ligands in 1 with $\mathrm{PPh}_{2} \mathrm{Me}$, show intrinsically different absorption spectra. As depicted in Figure 6, the $\mathrm{S}_{0}-\mathrm{S}_{1}$ absorption spectral features are quite different among $\mathbf{6}-\mathbf{8}$, in which the peak wavelengths of $398 \mathrm{~nm}(\mathbf{6}$, shoulder), $366 \mathrm{~nm}(7)$, and $360 \mathrm{~nm}(\mathbf{8})$ appear to be far red-shifted with respect to that of $\mathbf{1 - 4}(320 \mathrm{~nm})$ and $\mathbf{5}(305 \mathrm{~nm})$ (see Table 1 for comparison). The results showed good consistence with the general trend of photophysical properties that occurred after phosphine substitution. ${ }^{27}$ Moreover, supported by their extinction coefficients of $<3000 \mathrm{M}^{-1} \mathrm{~cm}^{-1}$, the corresponding $\mathrm{S}_{0} \rightarrow \mathrm{S}_{1}$ transition for $\mathbf{6}-\mathbf{8}$ can be

(27) Li, E. Y.; Cheng, Y.-M.; Hsu, C.-C.; Chou, P.-T.; Lee, G.-H.; Lin, I.-H.; Chi, Y.; Liu, C.-S. Inorg. Chem. 2006, 45, 8041. tentatively assigned to ${ }^{1}$ MLCT mixed, in part, with the ${ }^{1} \pi \pi^{*}$ transition in character. This assignment is considerably different from that of $\mathbf{1 - 5}$, in which a $\pi \pi^{*}$ ILCT is dominant for the lowest lying transition. Such a salient difference lies in the relative stronger $\sigma$-donating and less efficient $\pi$ accepting properties of $\mathrm{PPh}_{2} \mathrm{Me}$ in $\mathbf{6}-\mathbf{8}$, which pushes up the $\mathrm{d}_{\pi}$ orbital of Os(II) metal center and accordingly increases the associated HOMO energy level as well as the ${ }^{1}$ MLCT contribution. ${ }^{24}$

Furthermore, the $S_{0} \rightarrow S_{1}$ transition energy tends to be increased in the order of $\mathbf{6}<\mathbf{7}<\mathbf{8}$, the results of which can be rationalized by the fact that the remaining $\mathrm{CO}$ possesses a stronger $\pi$-accepting strength than that of $\mathrm{PPh}_{2}$ Me. As opposed to $\mathbf{7}$ and $\mathbf{8}$, in which the unique $\mathrm{CO}$ ligand is located opposite to the pyrazolate moiety, the $\mathrm{CO}$ ligand of $\mathbf{6}$ is in the trans position with respect to the pyridyl fragment. Since pyrazolate and pyridyl moieties, to a certain extent, determine the HOMO and LUMO energy levels, respectively (vide supra), the decrease of the electron density trans to CO such as pyridyl (LUMO in 6) and pyrazolate fragment (HOMO in $\mathbf{7}$ and 8 ) should lead to a decrease (increase) of the $S_{0} \rightarrow S_{1}$ energy gap for 6 (7 and 8 ), rationalizing the lowest $S_{0}-S_{1}$ energy gap for $\mathbf{6}$. Moreover, as for 7 versus $\mathbf{8}$, realizing that both $\pi$-accepting $\mathrm{PPh}_{2} \mathrm{Me}$ are located at the mutually trans disposition in $\mathbf{7}$, a lesser degree of $\pi$-back-donation from the Os(II) metal center to trans-disposed phosphines is thus anticipated. Accordingly, both the better $\sigma$-donation from phosphine ligands and the poor competition of the $\mathrm{d}_{\pi}$-electron in $\mathbf{7}$ would increase the relative electron density at the metal center, giving rise to a lower $\mathrm{S}_{0}-\mathrm{S}_{1}$ gap.

As for the luminescence properties, owing to its significant $\mathrm{O}_{2}$ quenching and/or the rather long radiative lifetime of tens to hundreds of microseconds (see Table 1), the origin of emission for the titled compounds being ascribed to phosphorescence is unambiguous. In a good correlation with the trend of absorption spectra, independent of the axial anions, complexes 1-4 all exhibit the expected unique blue emission with peak wavelengths at $\sim 445-460 \mathrm{~nm}$ in acetonitrile, while $\mathbf{5}$ revealed a significant blue shift with respect to that of 1-4 due to the replacement of pyrazolate by triazolate moiety. The similar trend for both $\mathrm{S}_{0} \rightarrow \mathrm{S}_{1}$ (absorption) and $\mathrm{T}_{1} \rightarrow \mathrm{S}_{0}$ (phosphorescence) transitions leads us to conclude that the frontier orbitals involved in the $\mathrm{S}_{0}-\mathrm{T}_{1}$ transition must be the same as that of $S_{0}-S_{1}$, i.e. largely incorporating the azolate (HOMO) pyridyl (LUMO) $\pi \pi^{*}$ transition. Qualitatively, prompt evidence of this viewpoint is given by the distinct vibronic progression of the phosphorescence in 1-5 
(Figure 5), a phenomenon symbolizing the transition dominated by the intraligand $\pi \pi^{*}$ character.

Another interesting point is that complexes $\mathbf{1}$ and $\mathbf{5}$, bearing tfa anions, exhibit much higher emission yield (1, $\mathrm{QY}=0.26 ; \mathbf{5}, \mathrm{QY}=0.27$ ) than those of $\mathbf{2 - 4}$, bearing halides. With emission quantum yield and observed lifetime provided, both radiative and nonradiative decay rate are also deduced and listed in Table 1. The radiative decay rates, $k_{\mathrm{r}}$, calculated as $\mathbf{1}\left(1.8 \times 10^{3} \mathrm{~s}^{-1}\right), \mathbf{2}\left(1.2 \times 10^{3} \mathrm{~s}^{-1}\right), \mathbf{3}(1.5 \times$ $\left.10^{3} \mathrm{~s}^{-1}\right), \mathbf{4}\left(3.0 \times 10^{2} \mathrm{~s}^{-1}\right)$, and $\mathbf{5}\left(5.8 \times 10^{3} \mathrm{~s}^{-1}\right)$ are rather small, manifesting their dominant $\pi \pi^{*}$ origin. Theoretically, the smaller radiative decay rate corresponds to less allowed $\mathrm{S}_{0}-\mathrm{T}_{1}$ transition. With application of the first-order approximation, it also implies the reduction of the spin-orbit coupling integral, most likely from the lesser $\mathrm{d}_{\pi}$ contribution of the heavy metal atom..$^{28}$ In other words, compared with the examples documented in our previous studies, ${ }^{23,29}$ these results lead to a proposal involving much reduced MLCT contribution for complexes $\mathbf{1 - 5}$ in the lowest energy triplet manifold $\mathrm{T}_{1}$.

While the radiative decay rates among $\mathbf{1 - 5}$ are on the same order of magnitude, the main difference in emission quantum yield lies in the more than 1 order of magnitude difference in the nonradiative decay rate $k_{\mathrm{nr}}$, namely $5.2 \times$ $10^{3} \mathrm{~s}^{-1}(\mathbf{1})$ and $1.6 \times 10^{4} \mathrm{~s}^{-1}(\mathbf{5})$, compared to the halidesubstituted complexes; cf. $4.0 \times 10^{4} \mathrm{~s}^{-1}(\mathbf{2}), 4.8 \times 10^{5} \mathrm{~s}^{-1}$ (3), and $3.3 \times 10^{6} \mathrm{~s}^{-1}(\mathbf{4})$. Due to the high-energy emission, we tentatively rationalize the results by the weakening of the Os(II)-halide bonding upon excitation, such that the excited-state potential energy surface (PES) tends to be shallower than those of tfa-anchored complexes $\mathbf{1}$ and $\mathbf{5}$. The net result not only destabilizes the molecular framework but also increases the radiationless deactivation process plausibly due to the resulting shallow PES in the $T_{1}$ state that may intersect with PES of the ground state. A parallel support is also given by the correlation between halide complexes and $k_{\mathrm{nr}}$, which shows a tendency of decreasing the bond strength in the order of $\mathrm{Os}(\mathrm{II})-\mathrm{Cl}>\mathrm{Os}(\mathrm{II})-\mathrm{Br}>\mathrm{Os}(\mathrm{II})-\mathrm{I}$, corresponding to the increase of $k_{\mathrm{nr}}$ in a trend of $\mathbf{2}<\mathbf{3}<\mathbf{4}$.

Figure 6 also reveals the emission spectra of 6 and 7 in degassed acetonitrile solution at RT. In good agreement with the difference in their absorption spectra, due to the lowering of LUMO by the $\pi$-accepting effect exerted by the CO ligand, the phosphorescence in $\mathbf{6}$ is red-shifted by $\sim 30 \mathrm{~nm}$ with respect to that of $\mathbf{7}$. Attempts to acquire emission of $\mathbf{8}$ unfortunately failed. Taking account of the instrument sensitivity, the upper limit of QY for $\mathbf{8}$ is estimated to be $10^{-4}$. Moreover, compared with those of $\mathbf{1}-\mathbf{5}$, a salient difference in the spectral feature can be promptly pointed out for both $\mathbf{6}$ and $\mathbf{7}$, in which the phosphorescence spectrum is broadened and lacks vibronic progression, a feature that manifests the dominance of the ${ }^{3}$ MLCT character. ${ }^{30}$ The ${ }^{3}$ MLCT-enhancing $\mathrm{T}_{1}-\mathrm{S}_{0}$ transition can be firmly supported by the deduced radiative decay rate constant of $8.3 \times 10^{4}$

(28) Yutaka, T.; Obara, S.; Ogawa, S.; Nozaki, K.; Ikeda, N.; Ohno, T.; Ishii, Y.; Sakai, K.; Haga, M.-A. Inorg. Chem. 2005, 44, 4737.

(29) Chen, K.; Cheng, Y. M.; Chi, Y.; Ho, M. L.; Lai, C. H.; Chou, P. T. Peng, S. M.; Lee, G. H. Chem. Asian J. 2007, 2, 155. $\mathrm{s}^{-1}$ and $8.6 \times 10^{4} \mathrm{~s}^{-1}$ for 6 and 7 , respectively, which is greater than that of $\mathbf{1}-\mathbf{5}$ by more than 1 order of magnitude (see Table 1).

In brief, for the titled complexes 1-8, the trend of energy gap, measured by either absorption $\left(\mathrm{S}_{0}-\mathrm{S}_{1}\right)$ or phosphorescence $\left(\mathrm{S}_{0}-\mathrm{T}_{1}\right)$, and the contribution of types of frontier orbitals can be well explained by the electronic properties and relative positions of the axial anion. Nevertheless, a puzzle pending resolution lies in the extremely weak (QY $\ll 0.01$ ) for $\mathbf{6}-\mathbf{8}$ despite their great ${ }^{3} \mathrm{MLCT}$ contribution, i.e., a consequence of the short radiative decay time. Accordingly, the nonradiative decay rate, $k_{\mathrm{nr}}$, for $\mathbf{6}$ and $\mathbf{7}$ was also deduced and listed in Table 1 . As for $\mathbf{1 - 5}$ with $\pi \pi^{*}$ dominance versus $\mathbf{6}$ and $\mathbf{7}$ with great MLCT contribution, the difference in $k_{\mathrm{nr}}$ of more than 2 orders of magnitude is much greater than the corresponding difference in $k_{\mathrm{r}}$. In other words, though $k_{\mathrm{r}}$ is significantly increased in $\mathbf{6}$ and $\mathbf{7}$ by promoting the MLCT contribution, the accompanied $k_{\mathrm{nr}}$ increases as well with an even more pronounced increment. Note Angelis et al. ${ }^{31}$ have recently studied some phosphorescent $\operatorname{Ir}(\mathrm{III})$ complexes for OLED applications and the results revealed a perfectly linear correlation between the nonradiative deactivation rate constant and energy-gap law. ${ }^{32}$ Generally, the energy-gap law works well under the circumstance of a large difference in energy gap and perhaps the same excited-state characters, such as pure $\pi \pi^{*}$. In contrast, in this study, we noticed that we could not obtain a linear proportional relation for $\ln \left(k_{\mathrm{nr}}\right)$ versus the emission energies among the titled Os(II) complexes. This "unusual observation" may actually be normal if light can be shed on the fundamental basis. The radiative decay rate constant is proportional to the electronic coupling matrix and the Franck-Condon weighed density of the state expressed as

$$
\begin{aligned}
k_{\mathrm{r}} \propto\left|\left\langle\mathrm{T}_{1} \Phi_{\mathrm{T}_{1} 0}\left|H_{\mathrm{er}}\right| \mathrm{S}_{0} \Phi_{\mathrm{S}_{0} m}\right\rangle\right|^{2}=\left|\left\langle\mathrm{T}_{1}\left|H_{\mathrm{er}}\right| \mathrm{S}_{0}\right\rangle\right|^{2} \mid & \\
\left.\left\langle\Phi_{\mathrm{T}_{1} 0} \mid \Phi_{\mathrm{S}_{0} m}\right\rangle\right|^{2} & =\left|\left\langle\mathrm{T}_{1}\left|H_{\mathrm{er}}\right| \mathrm{S}_{0}\right\rangle\right|{ }^{2} \mathrm{FC}_{\mathrm{T}_{1} 0}, \mathrm{~S}_{0} m
\end{aligned}
$$

where $H_{\text {er }}$ denotes the electric dipole operator created by the electric magnetic field. $\mathrm{FC}_{\mathrm{T}_{1} 0, \mathrm{~S}_{0} m}$ specifies the FranckCondon overlap factor between the vibrational wave function $(\Phi)$ of $\mathrm{T}_{1}$ at the vibrational quanta of $n=0$ and that of $\mathrm{S}_{0}$ at $n=m$. Note that $\mathrm{S}_{0}$ and $\mathrm{T}_{1}$ in eq 3 only denote the electronic wave function. In addition, to simplify the discussion, instead of the summation of entire vibrational states in $\mathrm{S}_{0}$, only one vibronic state, i.e., $m$, in $\mathrm{S}_{0}$ is considered for the transition.

In another approach, using a first-order approximation, the radiationless decay constant $k_{\mathrm{nr}}$ can be expressed as

(30) (a) Tung, Y.-L.; Chen, L.-S.; Chi, Y.; Chou, P.-T.; Cheng, Y.-M.; Li, E. Y.; Lee, G.-H.; Shu, C.-F.; Wu, F.-I.; Carty, A. J. Adv. Funct. Mater. 2006, 16, 1615. (b) Field, J. S.; Haines, R. J.; Ledwaba, L. P.; McGuire, R.; Munro, O. Q., Jr.; Low, M. R.; McMillin, D. R. Dalton Trans. 2007, 192.

(31) Angelis, F. D.; Fantacci, S.; Evans, N.; Klein, C.; Zakeeruddin, S. M.; Moser, J.-E.; Kalyanasundaram, K.; Bolink, H. J.; Gra1tzel, M.; Nazeeruddin, M. K. Inorg. Chem. 2007, 46, 5989.

(32) (a) Kober, E. M.; Caspar, J. V.; Lumpkin, R. S.; Meyer, T. J. J. Phys. Chem. 1986, 90, 3722. (b) Henry, B. R.; Siebrand, W. In Organic Molecular Photophysics; Birks, J. B., Ed.; Wiley: New York, 1973; Vol. 1, Chapter 4. (c) Freed, K. F.; Jortner, J. J. Chem. Phys. 1970 52, 6272. (d) Bixon, M.; Jortner, J. J. Chem. Phys. 1968, 48, 715. 
Cheng et al.

$$
\begin{array}{r}
k_{\mathrm{nr}} \propto \frac{4 \pi^{2} \rho_{E}}{h^{2}}\left|\left\langle\mathrm{~T}_{1} \Phi_{\mathrm{T}_{1} 0}\left|H_{\mathrm{nr}}\right| \mathrm{S}_{0} \Phi_{\mathrm{S}_{0}}(E)\right\rangle\right|^{2}=\frac{4 \pi^{2} \rho_{E}}{h^{2}}\left|\left\langle\mathrm{~T}_{1}\left|H_{\mathrm{nr}}\right| \mathrm{S}_{0}\right\rangle\right|^{2} \mid \\
\left.\left\langle\Phi_{\mathrm{T}_{1} 0} \mid \Phi_{\mathrm{S}_{0}}(E)\right\rangle\right|^{2}=\frac{4 \pi^{2} \rho_{E}}{h^{2}}\left|\left\langle\mathrm{~T}_{1}\left|H_{\mathrm{nr}}\right| \mathrm{S}_{0}\right\rangle\right|^{2} \mathrm{FC}_{\mathrm{T}_{1} 0, \mathrm{~S}_{0}(E)}(4)
\end{array}
$$

where $H_{\mathrm{nr}}$ is any nuclear kinetic energy operator that induces the jump between two PES's. ${ }^{32}$

$\mathrm{S}_{0}$ and $\mathrm{T}_{1}$ in eq 4 only denote the electronic wave function. $E$ in $\Phi_{\mathrm{S}_{0}}(E)$ specifies the vibrational energy between the zero point energies of $\mathrm{T}_{1}$ and $\mathrm{S}_{0}$. For nonlinear, polyatomic molecules such as the titled Os(II) complexes, due to the $3 n$ -6 degrees of vibrational freedom, there should be a large number of $\Phi_{\mathrm{S}_{0}}(E)$ states being isoenergetic with respect to $\Phi_{\mathrm{T}_{1} 0}$, which may be further broadened by solvent perturbation, merging into a continuum of state density $\rho_{E} . \mathrm{FC}_{\mathrm{T}_{1} 0, \mathrm{~S}_{0}(E)}$ is the Franck-Condon overlap factor, which describes the overlap of the continuum of vibrational states $\Phi_{\mathrm{S}_{0}}(E)$ with respect to $\Phi_{\mathrm{T}_{1} 0}$.

One can thus view the Franck-Condon factor as a vertical transition in $k_{\mathrm{r}}$, while it is a horizontal transition in $k_{\mathrm{nr}}$. The electronic coupling factors $\left|\left\langle\mathrm{T}_{1}\left|H_{\mathrm{er}}\right| \mathrm{S}_{0}\right\rangle\right|^{2}$ and $\left|\left\langle\mathrm{T}_{1}\left|H_{\mathrm{nr}}\right| \mathrm{S}_{0}\right\rangle\right|^{2}$ for $k_{\mathrm{r}}$ and $k_{\mathrm{nn}}$, respectively, involve the wave functions of initial $\left(\mathrm{T}_{1}\right)$ and final $\left(\mathrm{S}_{0}\right)$ electronic states, such that the radiationless transition is subject to the same multiplicity selection rules as the radiative transition..$^{33}$ In other words, the stronger $\mathrm{S}_{0}-\mathrm{T}_{1}$ mixing due to the greater ${ }^{3} \mathrm{MLCT}$ contribution, ${ }^{34}$ in parallel, leads to an increase of both $k_{\mathrm{r}}$ and $k_{\mathrm{nr}}$. In the cases of $6-\mathbf{8}$, a further increase of the radiationless transition perhaps can be qualitatively rationalized by the introduction of $\mathrm{PPh}_{2} \mathrm{Me}$, in which both methyl and phenyl torsional motions should drastically increase the density of the isoenergic states $\sigma$ in $k_{\mathrm{nr}}$ and hence lead to an increase of the radiationless deactivation rate constants. Moreover, the complexes $\mathbf{6}$ and $\mathbf{7}$ are akin to the hydride complexes of formula $\left[\mathrm{Os}(\mathrm{H})(\mathrm{fptz})\left(\mathrm{PPh}_{2} \mathrm{Me}\right)_{2}(\mathrm{CO})\right]$ reported in the literature. ${ }^{22}$ Yet the quantum yield for the hydride complexes is orders of magnitude higher than that of $\mathbf{6}$ and $\mathbf{7}$ with the tfa ligand. These data indicate that the current tfa complexes 6 and $\mathbf{7}$ possess much larger nonradiative rates than those found for the aforementioned hydride derivatives $\left((0.1-2.5) \times 10^{6}\right.$ $\mathrm{s}^{-1}$ ). This discrepancy can be tentatively rationalized by the difference in frontier orbitals involved in the low-lying transitions. Unlike the hydride complexes, as shown in Figure 8, the HOMO for $\mathbf{6}$ and $\mathbf{7}$ has a substantial contribution from the tfa ligand. As the $\mathrm{T}_{1} \rightarrow \mathrm{S}_{0}$ mainly involves LUMO $\rightarrow$ HOMO transition, the deactivation pathways should be subject to the associated tfa motions, and the quenching of emission is expected to be significant due to the relatively weak bonding to the tfa ligand.

\section{Theoretical Approaches}

Supplementary supports of the above-mentioned results and rationalization are rendered via the computational

(33) Birks, J. B. Photophysics of Aromatic Molecules; Wiley-Interscience: London and Colchester, U.K., 1970; p 151.

(34) (a) Azumi, T.; Miki, H. Top. Curr. Chem. 1997, 191, 1. (b) Solomon, E. I.; Lever, A. B. P. Inorganic Electronic Structure and Spectroscopy; John Wiley \& Sons: New York, 1999; Vol. I, Chapter 1.

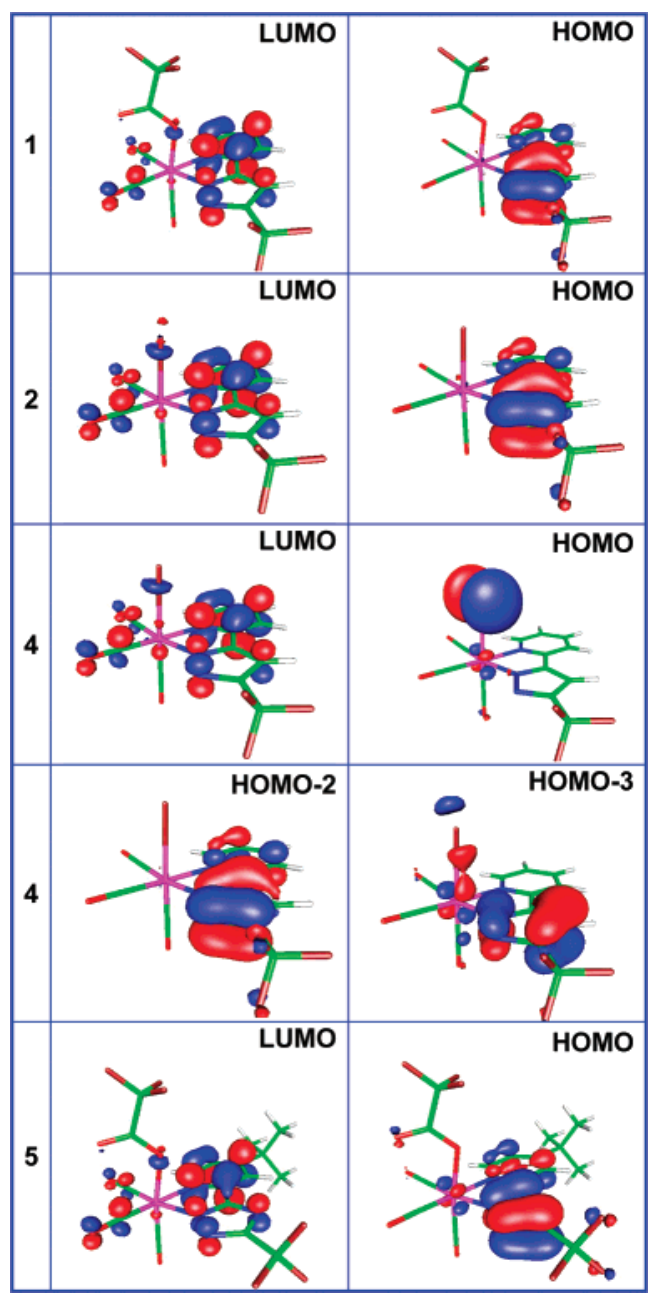

Figure 7. Highest occupied (HOMO) and lowest unoccupied (LUMO) molecular orbitals of complexes $\mathbf{1 , 2 , 4}$, and $\mathbf{5}$. Note that the frontier orbitals of bromide complex $\mathbf{3}$ were omitted because they appeared to be quite similar to those of chloride derivative $\mathbf{2}$.

approaches. Theoretical confirmation of the underlying basis for the photophysical properties of the studied complexes was provided by the density functional theory (DFT) MO calculations. With the use of the TD-B3LYP method, incorporating the B3LYP/LANL2DZ and 6-31G* optimized geometry, the vertical (i.e., Franck-Condon) excitation energy from the ground-state to low lying excited states was calculated. For validation of the theoretical method adopted here, comparisons between the optimized geometrical parameters and X-ray data for complexes $\mathbf{1}$ and $\mathbf{6}-\mathbf{8}$ are listed in the Supporting Information. The calculated structures are found to be in good agreement with experimental data. For example, in complex 1, the values of $\mathrm{Os}-\mathrm{O}(\mathrm{tfa}), \mathrm{Os}-\mathrm{C}(\mathrm{car}-$ bonyl) and $\mathrm{Os}-\mathrm{N}$ (azolate and pyridine) distances of 2.107 , $1.916-1.949,2.077$, and $2.174 \AA$ are calculated, to be comparable with experimental values of $2.098,1.907-1.962$, 2.061 , and $2.140 \AA$, respectively. Figure 7 depicts the features of the lowest unoccupied (LUMO) and the highest occupied (HOMO) frontier orbitals for complexes $\mathbf{1}, \mathbf{2}, \mathbf{4}$, and $\mathbf{5}$, which are mainly involved in the lower lying transitions. Note that the HOMO-2 and HOMO-3 orbitals were added to complex 4 because the orbitals mainly involved in the $\mathrm{S}_{0} \rightarrow \mathrm{T}_{1}$ transition are both HOMO-2 and HOMO-3 instead of 


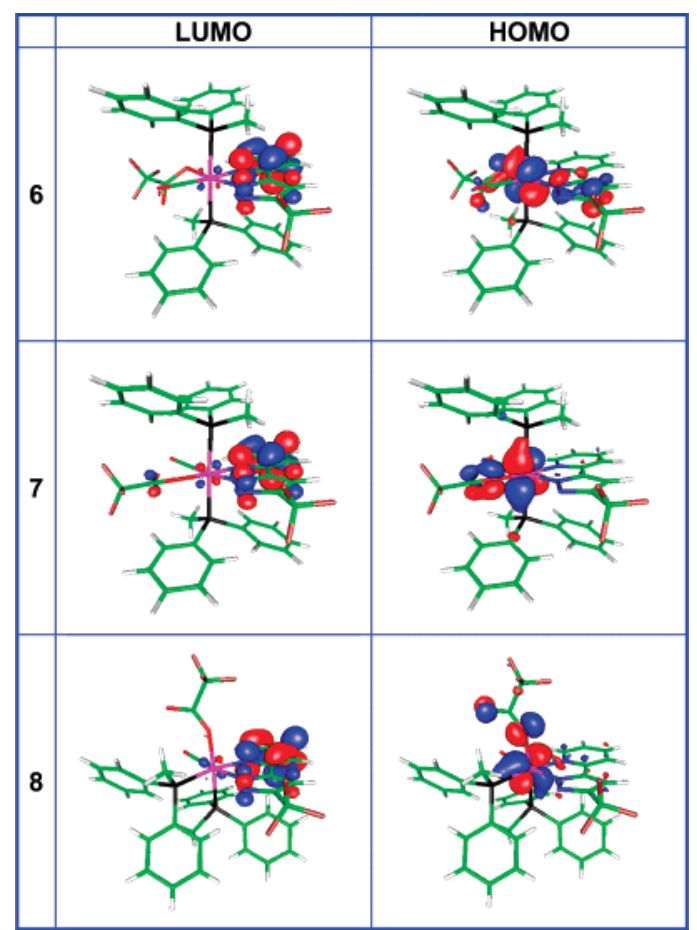

Figure 8. Highest occupied (HOMO) and lowest unoccupied (LUMO) molecular orbitals of complexes $\mathbf{6}-\mathbf{8}$.

HOMO. The descriptions and the energy gaps of all complexes are listed in Table 2. Obviously, the calculated $\mathrm{S}_{1}$ and $\mathrm{T}_{1}$ energy levels for the titled complexes are qualitatively consistent with those $0-0$ onsets of the absorption $\left(S_{1}\right)$ and phosphorescence $\left(\mathrm{T}_{1}\right)$ spectra. Thus, the theoretical level adopted here should be suitable for studying the photophysical properties of these complexes in a qualitative manner. Deviation of current theoretical approach from the experimental results may plausibly be explained by the negligence of solvation effects in the gas phase ab initio approach.

On the basis of the frontier orbital analyses, the lowest singlet and triplet excited states for these complexes are dominated by ILCT ( $\pi \pi^{*}$, azolate $\rightarrow$ pyridyl moiety). In other words, one can also envisage that the HOMO's of 1-5 are apparently much less located at the central Os(II) atom, leading to very small MLCT contribution. For example, results of the calculation show $\leq 1 \%$ MLCT contribution for 1-5, consistent with the conclusion drawn from the experimental results, i.e., the emission bands with vibronic progressions and rather long radiative decay time. The calculated energy gap is also consistent with experimental results, revealing a trend of similar $S_{0}-S_{1}$ and $S_{0}-T_{1}$ energy gaps for 1-4, while a significant blue shift in both was resolved for 5. Moreover, the HOMO of $\mathbf{4}$ showed significant contribution from the axial iodide substituent, which implicated the coexistence of the so-called halide-to-ligand charge transfer (XLCT) transition documented in the literature. ${ }^{35}$ However, due to the poor overlap between the $\mathrm{p}_{\pi}$ orbital of iodide and the $\pi^{*}$ orbital of the fppz chromophore, the transition dipole between the iodide $\mathrm{p}_{\pi}$ and the ligand $\pi^{*}$ orbitals should be small.

Finally, as depicted in Figure 8, the lowest lying transitions for complexes 6-8 are apparently dominated largely by MLCT mixed with $\pi \pi^{*}$ and LLCT transitions in character. ${ }^{10}$ As compared to complexes 1-5 (MLCT $\sim 1 \%$ ), the much larger extents of the calculated MLCT contributions of $\mathbf{6}$ $(55 \%)$ and $7(58 \%)$ are unambiguously responsible for the relatively much shorter radiative lifetime due to the direct involvement of MLCT in spin-orbit coupling. An equally important result is that the calculation also correctly predicts that the $\mathrm{S}_{0}-\mathrm{S}_{1}$ ( or $\mathrm{S}_{0}-\mathrm{T}_{1}$ ) energy gap is in the order of $\mathbf{8}>$ $7>6$, giving firm support for our early explanation (vide supra).

\section{Conclusion}

In summary, Os complexes with single $\pi$-chromophore were designed and synthesized in an aim to attain optimum blue phosphorescence. Our proposal of confining the excitation energy seems to work well upon anchoring of a single,

Table 2. Calculated Excitation Properties of the Lowest Lying Excited States of Complexes 1-8 ${ }^{a}$

\begin{tabular}{|c|c|c|c|c|c|}
\hline compd & state & $\lambda(\mathrm{nm})$ & $f$ & assignment & contents \\
\hline \multirow[t]{2}{*}{1} & $\mathrm{~T}_{1}$ & 438.1 & $\sim 0$ & HOMO $\rightarrow$ LUMO $(+94 \%) ;$ HOMO-1 $\rightarrow$ LUMO $(+7 \%)$ & $\mathrm{MLCT} \leq 1 \%$ \\
\hline & $\mathrm{S}_{1}$ & 343.8 & 0.0553 & HOMO $\rightarrow$ LUMO $(+87 \%) ;$ HOMO-1 $\rightarrow$ LUMO $(+12 \%)$ & MLCT $\leq 1 \%$ \\
\hline \multirow[t]{2}{*}{2} & $\mathrm{~T}_{1}$ & 436.5 & $\sim 0$ & $\mathrm{HOMO} \rightarrow \mathrm{LUMO}(+92 \%) ; \mathrm{HOMO} \rightarrow \mathrm{LUMO}+3(9 \%)$ & MLCT $\sim 1.5 \%$ \\
\hline & $\mathrm{S}_{1}$ & 346.5 & 0.042 & HOMO $\rightarrow$ LUMO $(+71 \%) ;$ HOMO- $1 \rightarrow$ LUMO $(+18 \%)$ & $\mathrm{MLCT} \sim 3 \%$ \\
\hline \multirow[t]{3}{*}{3} & $\mathrm{~T}_{1}$ & 437.5 & $\sim 0$ & $\mathrm{HOMO} \rightarrow \mathrm{LUMO}(+85 \%) ; \mathrm{HOMO} \rightarrow \mathrm{LUMO}+3(+10 \%)$ & MLCT $\leq 1 \%$ \\
\hline & $\mathrm{S}_{1}$ & 362.0 & 0.0045 & $\begin{array}{l}\text { HOMO- } 1 \rightarrow \text { LUMO }(+64 \%) ; \text { HOMO } \rightarrow \text { LUMO }(+23 \%) ; \\
\text { HOMO- } 2 \rightarrow \text { LUMO }(6 \%)\end{array}$ & MLCT $\sim 12 \%$ \\
\hline & $\mathrm{S}_{3}$ & 345.0 & 0.0477 & $\begin{array}{l}\text { HOMO } \rightarrow \text { LUMO }(+54 \%) ; \text { HOMO- } 2 \rightarrow \text { LUMO }(+17 \%) ; \\
\text { HOMO- } 1 \rightarrow \text { LUMO }(10 \%) ; \text { HOMO-3 } \rightarrow \text { LUMO }(8 \%)\end{array}$ & MLCT 3.6\% \\
\hline \multirow[t]{3}{*}{4} & $\mathrm{~T}_{1}$ & 436.4 & $\sim 0$ & $\begin{array}{l}\text { HOMO- } \rightarrow \text { LUMO }(+85 \%) ; \text { HOMO- } 2 \rightarrow \text { LUMO }+3 \\
(+11 \%) ; \text { HOMO- } 3 \rightarrow \text { LUMO }(+10 \%)\end{array}$ & $\mathrm{MLCT} \leq 1 \%$ \\
\hline & $\mathrm{S}_{1}$ & 392.4 & 0.0004 & HOMO $\rightarrow$ LUMO $(+92 \%)$ & MLCT $\sim 13 \%$ \\
\hline & $\mathrm{S}_{3}$ & 347.9 & 0.0435 & HOMO-2 $\rightarrow$ LUMO $(+76 \%) ;$ HOMO-3 $\rightarrow$ LUMO $(9 \%)$ & MLCT $\leq 1 \%$ \\
\hline \multirow[t]{2}{*}{5} & $\mathrm{~T}_{1}$ & 403.0 & $\sim 0$ & $\begin{array}{l}\mathrm{HOMO} \rightarrow \mathrm{LUMO}(+89 \%) ; \mathrm{HOMO} \rightarrow \mathrm{LUMO}+2(+7 \%) ; \\
\mathrm{HOMO} \rightarrow \mathrm{LUMO}+3(+7 \%)\end{array}$ & $\mathrm{MLCT} \leq 1 \%$ \\
\hline & $\mathrm{S}_{1}$ & 329.3 & 0.0751 & $\mathrm{HOMO} \rightarrow$ LUMO $(+89 \%)$ & MLCT $\leq 1 \%$ \\
\hline \multirow[t]{2}{*}{6} & $\mathrm{~T}_{1}$ & 542.1 & $\sim 0$ & HOMO $\rightarrow$ LUMO $(+97 \%) ;$ HOMO-1 $\rightarrow$ LUMO $(+3 \%)$ & MLCT $\sim 55 \%$ \\
\hline & $\mathrm{S}_{1}$ & 505.9 & 0.0128 & $\mathrm{HOMO} \rightarrow \mathrm{LUMO}(+95 \%)$ & MLCT 54\% \\
\hline \multirow[t]{2}{*}{7} & $\mathrm{~T}_{1}$ & 524.6 & $\sim 0$ & $\mathrm{HOMO} \rightarrow$ LUMO $(+93 \%) ; \mathrm{HOMO} \rightarrow \mathrm{LUMO}+3(8 \%)$ & MLCT $\sim 58 \%$ \\
\hline & $\mathrm{S}_{1}$ & 470.2 & 0.0203 & HOMO $\rightarrow$ LUMO $(+77 \%) ;$ HOMO-1 $\rightarrow$ LUMO $(+17 \%)$ & MLCT 57\% \\
\hline \multirow[t]{2}{*}{8} & $\mathrm{~T}_{1}$ & 457.0 & $\sim 0$ & HOMO $\rightarrow$ LUMO $(+96 \%) ;$ HOMO- $4 \rightarrow$ LUMO $+1(+5 \%)$ & MLCT $\sim 45 \%$ \\
\hline & $\mathrm{S}_{1}$ & 399.5 & 0.0639 & HOMO $\rightarrow$ LUMO $(+89 \%) ;$ HOMO-1 $\rightarrow$ LUMO $(+3 \%)$ & MLCT $\sim 43 \%$ \\
\hline
\end{tabular}

${ }^{a}$ Notice that the oscillator strengths $(f)$ of the $S_{1}$ states of both complexes 3 and $\mathbf{4}$ were found to be trivial; therefore, another singlet excited state ( $\left.S_{3}\right)$ with a much more prominent oscillator strength was discovered and listed here. 


\section{Cheng et al.}

higher energy gap $\pi$-chromophore in the Os(II) complexes; consequently, the control of MLCT versus $\pi \pi^{*}$ (or LLCT) excited-state characters becomes much more straightforward. However, it is now necessary to mull over other adverse effects in that the localization of the excitation energy seems to be more facile to populate at certain vibrational modes with weak metal-ligand bond strength, i.e., with the shallow PES, inducing the unwanted radiationless transition. In addition, in comparison to the complexes with dual or multiple chromophores, axial anionic ligands play an important role for $\mathbf{1 - 8}$ in fine-tuning not only the energy gap but also the emission intensity, i.e., in manipulating the radiative versus the radiationless transition pathways. Our results clearly show that there is a tradeoff upon varying the parameters in an aim to tune both emission wavelength and intensity. Switching from dual or multiple chromophores to the single chromophore would localize the energy and consequently improve the emission bandwidth; it conversely may induce the radiationless transition due to confinement of the excitation energy in a local chromophore with certain weak bonding modes.

In yet another approach, increasing ${ }^{3} \mathrm{MLCT}$ is a general way to enhance the phosphorescence QY to find better OLED dopants, especially when the blue hue is to be paid much attention. However, it will also be subject to an increase of the radiationless transition due to a good $\mathrm{S}_{0}-\mathrm{T}_{1}$ mixing. Owing to the advantage of increased ${ }^{3} \mathrm{MLCT}$

(35) (a) Nieuwenhuis, H. A.; Stufkens, D. J.; Vlček, A., Jr. Inorg. Chem. 1995, 34, 3879. (b) Slageren, J. V.; Hartl, F.; Stufkens, D. J.; Martino, D. M.; Van Willigen, H. Coord. Chem. Rev. 2000, 208, 309. (c) Chen, Y.-L.; Lee, S.-W.; Chi, Y.; Hwang, K.-C.; Kumar, S. B.; Hu, Y.-H.; Cheng, Y.-M.; Chou, P.-T.; Peng, S.-M.; Lee, G.-H.; Yeh, S.-J.; Chen, C.-T. Inorg. Chem. 2005, 44, 4287. character in the excited-state and hence the shorter radiative lifetime in the OLED applications (vide supra), a strategic design may be needed to avoid the possible involvement of metal-centered $\mathrm{dd}^{*}, \sigma \pi^{*},{ }^{10}$ XLCT transitions as well as the incorporation of strong trans effect as the lowest lying transition that causes the shallow PES. This, in combination with a more rigid, robust structure to reduce the density of the accepting states, should provide a preliminary rule of thumb for attaining decent emission intensity, at least for the Os(II) relevant complexes bearing single chromophore. The optimization of such a strategic design can be previewed via the computational approach, such that the frontier orbitals involved in the lowest lying transition can be analyzed in a qualitative manner to increase the feasibility. We hope that the results and discussion presented here can make some contributions in the field for the development of all phosphorescent OLEDs. Especially, we believe that current progress in assembling and gaining photophysical backgrounds of the Os(II)-containing emissive molecules warrant a similar applicability to the related isoelectronic $\operatorname{Ir}($ III) materials and/or the square planar $\mathrm{Pt}(\mathrm{II})$ system with $\mathrm{d}^{8}$ configuration.

Acknowledgment. We thank the support from the following research grants: NSC 91-2119M-002-016; NSC 932752-M-002-002-PAE; 94-EC-17A-08-S1-042.

Supporting Information Available: X-ray crystallographic data files (CIF) for complexes $\mathbf{1}, \mathbf{6}, \mathbf{7}$, and $\mathbf{8}$ and a table showing the optimized geometrical parameters obtained from DFT calculation. This material is available free of charge via the Internet at http://pubs.acs.org.

IC7015269 